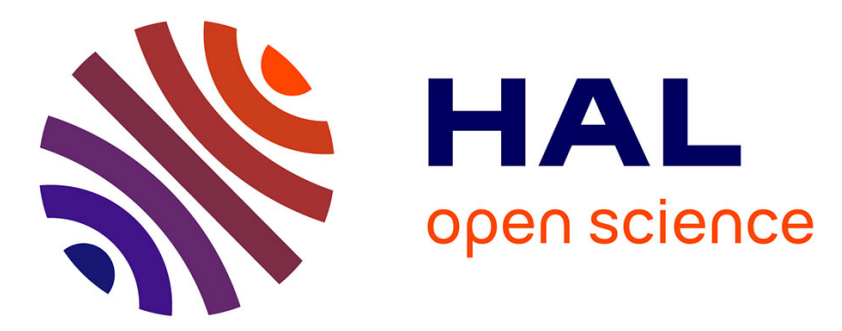

\title{
A Survey of Prostate Segmentation Methodologies in Ultrasound, Magnetic Resonance and Computed Tomography Images
}

Soumya Ghose, Arnau Oliver, Robert Marti, Xavier Llado, Joan C. Vilanova, Jordi Freixenet, Jhimli Mitra, Désiré Sidibé, Fabrice Mériaudeau

\section{To cite this version:}

Soumya Ghose, Arnau Oliver, Robert Marti, Xavier Llado, Joan C. Vilanova, et al.. A Survey of Prostate Segmentation Methodologies in Ultrasound, Magnetic Resonance and Computed Tomography Images. Computer Methods and Programs in Biomedicine, 2012. hal-00695557

\section{HAL Id: hal-00695557 https://hal.science/hal-00695557}

Submitted on 9 May 2012

HAL is a multi-disciplinary open access archive for the deposit and dissemination of scientific research documents, whether they are published or not. The documents may come from teaching and research institutions in France or abroad, or from public or private research centers.
L'archive ouverte pluridisciplinaire HAL, est destinée au dépôt et à la diffusion de documents scientifiques de niveau recherche, publiés ou non, émanant des établissements d'enseignement et de recherche français ou étrangers, des laboratoires publics ou privés. 


\title{
A Survey of Prostate Segmentation Methodologies in Ultrasound, Magnetic Resonance and Computed Tomography Images
}

\author{
Soumya Ghose $e^{\mathrm{a}, \mathrm{b}, *}$, Arnau Oliver ${ }^{\mathrm{a}, 1,2, * *}$, Robert Martí, Xavier Lladóa $^{\text {, }}$ \\ Joan C. Vilanova ${ }^{\mathrm{c}}$, Jordi Freixenet ${ }^{\mathrm{a}}$, Jhimli Mitra ${ }^{\mathrm{a}, \mathrm{b}}$, Desire Sidibe ${ }^{\mathrm{b}}$, Fabrice Meriaudeau ${ }^{\mathrm{b}}$ \\ ${ }^{a}$ Computer Vision and Robotics Group, University of Girona, Campus Montilivi, Edifici P-IV, 17071 Girona, Spain \\ ${ }^{b}$ Laboratoire Le2I - UMR CNRS 6306, Université de Bourgogne, 12 Rue de la Fonderie, 71200 Le Creusot, France \\ ${ }^{c}$ Girona Magnetic Resonance Center, Joan Maragall 26, 17002 Girona, Spain
}

\begin{abstract}
Prostate segmentation is a challenging task, and the challenges significantly differ from one imaging modality to another. Low contrast, speckle, micro-calcifications and imaging artifacts like shadow poses serious challenges to accurate prostate segmentation in transrectal ultrasound (TRUS) images. However in magnetic resonance (MR) images, superior soft tissue contrast highlights large variability in shape, size and texture information inside the prostate. In contrast poor soft tissue contrast between prostate and surrounding tissues in computed tomography (CT) images pose a challenge in accurate prostate segmentation. This article reviews the methods developed for prostate gland segmentation TRUS, MR and CT images, the three primary imaging modalities that aids prostate cancer diagnosis and treatment. The objective of this work is to study the key similarities and differences among the different methods, highlighting their strengths and weaknesses in order to assist in the choice of an appropriate segmentation methodology. We define a new taxonomy for prostate segmentation strategies that allows first to group the algorithms and then to point out the main advantages and drawbacks of each strategy. We provide a comprehensive description of the existing methods in all TRUS, MR and CT modalities, highlighting their key-points and features. Finally, a discussion on choosing the most appropriate segmentation strategy for a given imaging modality is provided. A quantitative comparison of the results as reported in literature is also presented.
\end{abstract}

Keywords:

Prostate gland segmentation methods, TRUS images, MR images, CT images.

\footnotetext{
${ }^{*}$ Corresponding Author

** Principal Corresponding Author

Email addresses: soumyaghose@gmail.com (Soumya Ghose), aoliver@eia.udg.edu (Arnau Oliver), marly@eia.udg.edu (Robert Martí), llado@eia.udg.edu (Xavier Lladó), jordif@eia.udg.edu (Jordi Freixenet), jhimlimitra@yahoo.com (Jhimli Mitra), desire.sidibe@u-bourgogne.fr (Desire Sidibe), fabrice.meriaudeau@u-bourgogne.fr (Fabrice Meriaudeau)

${ }^{1}$ Phone: (+34)972418878

${ }^{2}$ Fax: (+34) 972418259
} 


\section{Introduction}

Statistics from Cancer Research UK show that more than 338, 000 people are diagnosed with prostate cancer every year in Europe and 913,000 worldwide [1], posing prostate cancer as a major health problem. The highest rate of prostate cancer cases are diagnosed in USA, Australia, New Zealand, Western and Northern Europe, while the lowest rates are observed in South and Central Asia [1].

Primarily transrectal ultrasound (TRUS), magnetic resonance imaging (MRI) and computed tomography (CT) imaging are used in diagnosis, treatment, and follow-up of the prostate cancer. Figure 1 shows an example of a prostate obtained in each of these imaging techniques, where we can see that the information provided by each modality significantly differ from each other. The use of a particular modality depends on the clinical aim. For instance, MRI is primarily used in diagnostic and treatment planning for prostate diseases [2, 3], since it provides good soft tissue contrast and enables a better lesion detection and staging for prostate cancer. In addition, dynamic contrast enhanced MRI (DCE-MRI) aids in identifying malignant tissues from the diffusion rate of the contrast agent [4-6] and magnetic resonance spectroscopy aids in identifying malignant tissues from the relative concentration of different metabolites (like citrate, choline and creatine) [7]. On the other hand, TRUS is primarily used in determining prostate volume and in prostate biopsy due to the fact that it is an inexpensive, portable and real-time in nature [8]. Note from Figure 1(a) that TRUS images are characterized by speckle, shadow artifacts and low contrast [9] where the prostate gland can be often observed as a hypoechoic mass surrounded by a hyperechoic halo [10]. Finally, CT is generally used in prostate brachytherapy to determine the placement of the radioactive seeds and also to confirm the seed location post-procedure [7]. The high attenuation of the radioactive seed produces high intensity in CT images as could be visualized in Figure 1(c). Note, that distinguishing external and internal anatomy of prostate from CT images is difficult due to poor soft-tissue resolution. The main features associated with the different imaging modalities are summarized in Table 1.

In this article we will primarily focus on methods developed for prostate gland segmentation in TRUS, MR and CT images. Prostate segmentation from TRUS, MRI and CT plays a key role in different stages of clinical decision making process. For instance prostate volume, that can be directly determined from prostate gland segmentation, aids in diagnosis of benign prostate hyperplasia. The prostate boundary is utilized in different treatments of prostate diseases, like prostate brachytherapy, high intensity focused ultrasonography, in cryotherapy and in transurethral microwave therapy. Moreover, both prostate volume and contour are also useful in the follow up of prostate brachytherapy. In addition, prostate gland segmentation also facilitates multimodal image fusion for tumor localization in biopsy, minimally invasive ablative and radiation therapy. However, manual segmentation of the prostate is a tedious task, prone to inter and intra observer variability. Therefore, computerized schemes are currently being investigated to perform this task.

Three related surveys on prostate segmentation were published by Zhu et al. [11] in 2006, Noble et al. [12] in 2006, and Shao et al. [8] in 2003. Zhu et al. carried out a survey on computerized techniques developed for prostate cancer 
detection and staging, including not only prostate segmentation but also prostate staging, computerized visualization and simulation of prostate biopsy, volume estimation and registration between US and MR modalities. Noble et al. presented a survey on US segmentation methods developed for different organs (i.e. heart, breast, prostate) and for the detection of vascular diseases. Finally, Shao et al. presented a survey on prostate segmentation methodologies developed for TRUS images. Therefore, the surveys of Noble et. al. and Zhu et al. were done on a broader perspective and hence an exhaustive classification and discussion focused only on prostate segmentation methods was missing, while Shao et al. restricted their discussion to methodologies developed only for TRUS images.

This paper presents an up-to-date summary of the techniques developed for prostate segmentation in TRUS, MRI and CT modalities. We classify and review the different approaches found in the literature in order to show similarities and differences and further to extract advantages and drawbacks from the reviewed algorithms. To have an overall qualitative estimation of the performance of the different methods, we have grouped the methods according to their theoretical approach and have presented their evaluation metrics and degree of validation. Note that a quantitative comparison of different prostate segmentation methodologies is difficult in absence of public data sets, publicly available software, and standardized evaluation metrics.

In summary, we consider that the major contributions of this paper are:

- A new classification scheme grouping the surveyed methods on the basis of the theoretical approaches to the problem. Such classification is useful in highlighting the similarities and differences of the reviewed approaches.

- The inclusion of prostate segmentation approaches in all TRUS, MR and CT modalities in a single article. This is key aspect for finding links and differences between the different segmentation techniques, and it is even more important with the growing popularity of multi-modal prostate segmentation. Furthermore, to the best of our knowledge, for the first time a review on prostate segmentation methods developed in CT imaging is presented.

- A comparison of different segmentation methods based on their results are also presented.

- A discussion about choosing an appropriate segmentation method for a given imaging modality is carried out.

The outline of the paper is as follows. The state-of-the-art computer-aided prostate segmentation procedures are classified and presented in section 2. In section 3, validation and quantitative evaluation of the prostate segmentation in TRUS, MR and CT images are provided. Discussion on selection of an efficient prostate segmentation technique based on imaging modality is presented in section 4. Finally, the paper ends with conclusion and future trends.

\section{Prostate segmentation methods}

In this work, we classify the prostate segmentation methods according to the theoretical computational approach taken to solve the problem. We believe that such a classification successfully points out the key algorithmic similarities and dissimilarities, highlighting their strengths and weaknesses at the same time. We globally classify the methods 
into different strategies: contour and shape based, region based, supervised and un-supervised classification methods based, and hybrid methods. We further refine these groups to produce a more local classification schema. For instance, contour and shape based methods are further classified into edge, probabilistic filters and deformable models. The full taxonomy proposed in this paper is shown in Figure 2 that also presents the work that follow each strategy. Note that level sets methods appear under contour and shape based and region based methods. This is due to the fact that level sets can be guided by either boundary or region information.

We have grouped the prostate segmentation methods in four different groups, according to the information used to guide the segmentation. Broadly,

- Contour and shape based methods: These methods use prostate boundary/edge information to segment the prostate. Since often edge information is unreliable in TRUS and CT images and in the base and the apex region of the MR images, prior shape information is incorporated to provide better results.

- Region based methods: These methods use local intensity or statistics like mean and standard deviation in an energy minimization framework to achieve segmentation. The methods in this category primarily varies depending on the energy minimization framework. For example in atlas based methods a model of the prostate is created from manually segmented training images and intensity difference between the model and a new un-segmented image is minimized. In contrast, in region based level sets prior mean and standard deviation information of the prostate region from manually segmented images are used to maximize the distance between prostate and background regions depending on region based statistical moments and propagate an implicitly defined deformable model whose energy is minimized at the zone of convergence of the two regions.

- Supervised and un-supervised classification methods: These methods use features like intensity or higher dimensional features like filter responses to cluster and/or classify the image into prostate and background regions. The objective of such methods are to group similar objects together based on the feature vector. Unlike region based methods of energy minimization frameworks a thresholding scheme is used based on some proximity or distance measure to group similar objects together.

- Hybrid methods: The objective of the hybrid methods is to combine information from contour, shape, region and/or supervised or un-supervised classification information to segment the prostate. These methods are more robust to imaging artifacts and noise.

In the following subsections, the reviewed methods are described according to the presented taxonomy. Moreover, for each category, the approaches are grouped and described according to the imaging modalities: TRUS, MRI, and CT. Observe that this classification allows to easily see if the approaches belonging to one category are useful for segmenting the prostate in a given modality. 


\subsection{Contour and shape based segmentation}

Contour and shape based methods exploit contour features and shape information to segment the prostate. These methods can be categorized into edge based methods, probabilistic filters and deformable model segmentation techniques. Deformable model based techniques are further classified into active contour models, deformable meshes, active shape models, level sets and curve based segmentation. The following subsections discuss individually each of these categories.

\subsubsection{Edge based segmentation}

Extracting edges in an image using gradient filters like Prewitt, Robert, Sobel, Shen and Castan and Canny, is a popular practice in image processing. However, in presence of noise gradient filters often detect false edges and also the detected edges are often broken. Although computationally expensive edge linking algorithms have to be designed to produce connected edges, in most cases is necessary to combine edge based algorithms with intensity based and texture based information for accurate segmentation [13].

\section{TRUS}

Prostate segmentation based on edge information seems to be particularly difficult in TRUS images. Traditional edge detection filters fail to obtain accurate edges due to the low contrast, speckle and other imaging artifacts like shadow regions. To overcome these problems, Liu et al. [14] propose to use a radial bass relief representation of the prostate, which consists in superimposing the original image with a zoomed negative of the same. Kwoh et al. [15] used harmonics from the Fourier transform to reduce spurious edges of this representation. Other approaches aim to reduce the speckle from the original image. For instance, Aarnink et al. [16] used local standard deviation to identify homogeneous and heterogeneous regions in the image in a multi-resolution framework, and this information was considered for detecting the prostate boundary with more reliability. In contrast, Pathak et al. [17] reduced speckle by applying a stick filter based on the non-zero correlation value of speckle over large distances. The intensity value of the central pixel was replaced by the average of the intensity values in the horizontal, vertical and diagonal directions of a given size. The resulting image was further smoothed using an anisotropic diffusion filter. In the third stage, some basic prior knowledge of the prostate, such as shape and echo pattern, is used to detect the most probable edges describing the prostate. Finally, patient-specific anatomic information is integrated during manual linking of the detected edges to segment the prostate.

\section{MRI}

The use of typical edge detector operators in MR images can produce many false edges due to the high soft tissue contrast. Hence, Zwiggelaar et al. [18] used first and second order Lindeberg directional derivatives [19], in a polar coordinate system to identify the edges. An inverse transform of the longest curve selected after non maximal suppression of disconnected curves in the vertical direction was used to obtain the prostate boundary. On the other hand, Samiee et al. [20] used prior information of the prostate shape to refine the prostate boundary. Average gradient values obtained from a moving mask (guided by prior shape information) were used to trace out the prostate boundary. In 
a similar way, Flores-Tapia et al. [21] used a priori shape information of the prostate to trace out the boundary by the movement of a small mask on a feature space constructed from the product of the detail coefficients of the Haar wavelets in a multi-resolution framework.

\subsubsection{Probabilistic filtering}

Probabilistic filters like the Kalman filter [22], the probabilistic data association filter (PDAF) [23] and particle filters [24] have been successfully used to segment images. These methods model the boundary of an organ as a probabilistic trajectory of a moving object where the motion is governed by a dynamic model subject to a particular uncertainty. Segmentation algorithms based on probabilistic filters are fast as no optimization framework is necessary [25]. However, these methods may be sensitive to the initialization and the extension to 3D segmentation is complicated. Hence, to the best of our knowledge no method has been developed for 3D segmentation of the prostate in MR and in CT images.

\section{TRUS}

Abolmaesumi et al. [25] used PDAF to segment the prostate in TRUS images. The stick filter [17] was used to reduce speckle and enhance the contrast. The authors argued that the boundary of the prostate was given by a trajectory of an object whose motion was governed by a model from a finite set of known models at any given radius. The models differed in uncertainty levels and structures, and switched between the models depending on the Markov transitional probability [26]. The authors assumed that the acceleration could be modeled by Gaussian noise and the model produced a noisy version of the actual position of the particle. Each trajectory was associated with a Kalman filter and the output was combined with an interactive multiple model and PDAF to estimate the boundary location. On the other hand, as the prostate in TRUS images is characterized by a hypoechoic mass surrounded by hyperechoic perimeter [10], Sahba et al. [27] used median filtering followed by top hat and bottom hat transforms to effectively separate bright areas from dark regions trapping the characteristic feature. Binary thresholding followed by morphological filtering produced a smooth contour of the boundary. Subsequently, a Kalman filtering followed by a fuzzy inference produced the final prostate contour.

\subsubsection{Deformable model based segmentation}

Deformable model segmentation techniques are influenced by theories from geometry, physics and mathematical optimization. Geometry imposes constraints on the model shape, physical theories guide the evolution of the shape in space, and optimization theory guides the model to fit the available data [28]. Deformable models are often associated with internal and external energies. External energies propagate the deformable model towards the object boundary and internal energies preserve smoothness of the contours during deformation. Internal and external energies associated with a deformable model are combined and included in an energy minimization framework to segment anatomical structures by warping to the edges with minimum deformation away from their mean shape. The methods proposed in a deformable model framework may be broadly classified into active contour models, deformable mesh, 
active shape models, level sets and curve fitting.

\subsubsection{Active contour models}

The active contour model (ACM) or snake was initially developed by Kass et al. [29]. On initialization close to an edge, the active contour model evolves following the direction of the gradient in progressive deformation and stops at the edge. However, different external energies like balloon force [30], distance potential force [31] and gradient vector flow [32] have been proposed to improve the capture range of active contour.

\section{TRUS}

Considering low contrast in TRUS images, localization of true prostate edge to produce external energy is a real challenge. Knoll et al. [33] used maxima of a multi-scale dyadic wavelet to determine prostate edges. Balloon force was used as external force to deform a snake towards the maxima of the dyadic wavelet transform to segment the prostate in a multi-resolution framework. The form restricted contour deformation and its initialization by template matching are performed in a coarse to fine segmentation process based on a multiscale image edge representation containing the important edges of the image at various scales. To improve on the internal force of the ACM, Ladak et al. [34] used cubic interpolation between four points selected by the user to produce a discrete dynamic contour (DDC) [35]. Ding et al. [36] used a cardinal spline to construct the initial contour of the prostate from three or more manually selected points located in the prostate boundary. The final contour produced in one slice was used to initialize the neighboring slices. To improve on the capture range of the gradient force, Jendoubi et al. [37] used gradient vector flow [32] computed from the gradient map obtained using sobel and laplacian of Gaussian as external force to drive active contour towards the boundary of the prostate. Zaim et al. [38] used difference of Gaussian followed by non maximal suppression to detect dot patterns that were coherent with prostate tissue texture. An active contour constructed from manual delineations of prostate with dot pattern and gradient as external energy was used to segment the prostate.

\subsubsection{Deformable mesh}

Broadly, deformable meshes could be categorized into shape constrained deformable mesh or parametric deformable mesh. The methods included in the first category usually start dividing an initial manual segmentation in triangular and tetrahedral facets. Subsequently, similar to an ACM framework, the mesh deforms under the influence of internal and external forces to produce the desired segmentation. The objective of internal forces is to maintain a smooth surface while an external force drives the model towards the boundary of the organ. Often, the principal curvature of the surface is used as internal energy and the gradient is one of the most popular choices for external energy. However, gradient is usually combined with texture to improve the segmentation results. On the other hand, in the parametric deformable model, the deformable mesh is constructed on the basis of a three dimensional geometrical figure like a 
sphere, ellipsoid or a cube that has a close resemblance with the organ. Geometrical parameters are used for internal energy computation. Either gradient or texture or both are used as external forces to deform the mesh.

\section{TRUS}

To maintain the prostate shape, Ghanei et al. [39] used a shape constrained deformable mesh in a multi-resolution framework to achieve three dimensional segmentation of the prostate. Principal curvature of a surface from Todd and McLeod's method [40] was used as the internal force. The external force was computed from the expansion and the restoration model proposed by Rao and Ben-Arie [41]. A Gaussian noise model was assumed, while an edge was considered as a step function. An impulse response function was generated and applied to the volumetric data to generate the gradient. The gradient obtained in the process was used as the external force for mesh propagation for segmenting the prostate.

\subsubsection{Active shape model}

In absence of prior shape information, the final segmentation result of deformable models results often vary widely from the shape of the anatomical structure. Cootes et al. [42] proposed the active shape model (ASM) that worked in the deformable model framework maintaining the principal modes of shape variations of the anatomical structures under study. Principal modes of shape variations are identified by principal component analysis (PCA) of the point distribution models (PDM) [42] aligned to a common reference frame with generalized Procrustes analysis. Shape space is assumed to be Gaussian and is represented with a mean shape added to weighted principal modes of variations identified from PCA. With the initialization of the shape model, each landmark is searched within local vicinity to reach a better position with respect to the edges with a minimum displacement constraint that maintained the shape. Once all landmarks were displaced, scaling, rotation and translation parameters were chosen that minimized the distance between the deformed contour and the shape model. Prior shape information incorporated in active model makes it robust to noise and artifacts and produces improved segmentation results. In order to consistently set the corresponding landmarks automatically, the minimum descriptor length and Hill's algorithm [43] were proposed. The different methods primarily differed in the optimization framework and the feature space used for modeling the deformation.

\section{TRUS}

Shen et al. [44] used rotational invariant Gabor features computed with respect to the TRUS probe to characterize the prostate boundaries in multiple scales and multiple orientations. The Gabor features are further reconstructed to be invariant to the rotation of the ultrasound probe and incorporated in the prostate model as image attributes for guiding the deformable segmentation. The real and imaginary parts of Gabor features were used for smoothing and edge detection respectively. A hierarchical deformation strategy is then employed in which the model adaptively focuses on the similarity of different Gabor features at different deformation stages using a multiresolution technique form coarse to finer features to achieve segmentation. Similarly, Betrouni et al. [45] enhanced the prostate edge and reduced noise 
using a priori knowledge of the noise in TRUS images. An ASM was then used to produce the segmentation of the prostate. Hodge et al. [46] used the mean of manual segmentation from three experts to produce the ground truth value for prostate in TRUS images. An ASM was constructed from manually delineated contours after the reduction of noise using a median filter. The authors proposed to modify PDM of Cootes to generate all plausible shapes by dividing the prostate mid gland images into three regions and creating three plausible prostate shapes for each.

\section{MRI}

Cootes et al. [42] proposed to segment prostate in MR slices using the framework of ASM (they actually proposed prostate segmentation as one of the applications of their generic ASM model). Zhu et al. [47] proposed a hybrid of two and three dimensional ASMs to segment the prostate in MR data sets. A three dimensional ASM was built that represented the shape variance of the prostate. In each iteration, the three dimensional ASM was updated by the final search result of two dimensional segmentation. The authors claimed that, unlike pure 3D ASM, their hybrid ASM had a superior performance in sparse three dimensional data sets as 3D ASM built from sparse data was inefficient in detecting all possible modes of shape variations.

\section{CT}

The first attempt to use ASM to segment CT images of the prostate was done by Tang et al. [48], who used ASM to segment the prostate, but also the bladder and the rectum. In contrast, Feng et al. [49] used an ASM to segment only the prostate. Deviating from traditional ASM, the image correspondences were obtained by means of SIFT features [50]. Inter and intra patient specific ASM were built from manually delineated contours of the prostate.

\subsubsection{Edge based level sets}

The level sets framework introduced by Osher et al. [51] is a popular, powerful and efficient tool for medical image segmentation. This framework was developed to study curve propagation in higher dimensions. The level set is allowed to expand starting from a seed point in a direction normal to the curve surface that produces the segmented contour, with a speed inversely proportional to the intensity gradient. The evolution finally stops where the intensity difference is highest in a local neighborhood. Hence, the propagation of the curve in a level sets framework may help in finding an object boundary, and allows an efficient curve splitting and merging based on topological changes.

\section{TRUS}

Considering intensity heterogeneity of the prostate gland, it is difficult to segment prostate with traditional level set initialized on gray-scale images. Hence, Kachouie et al. [52] used Gaussian filtering followed by morphological filtering to classify the mid gland image into prostate and non prostate regions. An elliptical level set automatically initialized inside the prostate region was used to segment the prostate using first and second order moments of a 
Gaussian probability density function. The authors then used modified local binary patterns (LBP) to extract texture features of the prostate gland in TRUS images [53]. Gradient magnitude information of the modified LBP map was used as the external force to drive the elliptical level set to convergence, thereby segmenting the prostate.

\subsubsection{Curve fitting}

Parametric curves like splines, ellipses and Bezier curves are often used to segment prostate due to a close resemblance between the central gland of the prostate and an elliptical curve. Curve parameters are used as internal force and gradient as external force to deform the curve towards the prostate boundary.

\section{TRUS}

Hu et al. [54] used an ellipsoid, initialized from manual delineations of the limits of the axes, to produce 3D prostate segmentation. Ellipsoid warping using thin plate spline transformation was used to map the user selected six control points to the end of the semi major axis of the ellipsoid to ensure a better fitting. The deformation of the ellipsoid was influenced by the internal and external forces to produce the segmentation. In a similar way, Ding et al. [55] used a deformable super ellipse to just obtain an initial estimate of the prostate contour. Subsequently, the initial parameters of the super ellipse and gradient information of the image were jointly optimized to produce the final segmentation. To reduce propagation errors, a continuity constraint based on an autoregressive model was imposed on the initialization of the contour in new slices. Badiel et al. [56] also used an elliptical curve to segment the prostate. The ellipse was fitted through six user defined points. The deformation of the prostate was modeled with a sine function in the angular direction and with a Gaussian function in the radial direction. The warping function was built using these two functions to create an elliptical shape for the prostate. Finally, segmentation of the prostate was achieved by ellipse fitting to the prostate boundary obtained by interacting multiple modes PDAF [25] and reverse warping. In contrast to these works, Saroul et al. [57] used a tapered super ellipse to segment the prostate. The prostate gland was divided into eight octants and the intensities of each octant were modeled using a Rayleigh distribution. The tapered super ellipse was combined with the probability density functions of the intensities of the prostate and non prostate region in an energy optimization framework to segment the prostate region. Mahdavi et al. [58] used a similar tapered ellipsoid to segment the prostate. The authors used untapering and warping of the image to make the shape of the prostate elliptical. Probe center as well as the bottom, center, middle right, and bottom right of the prostate gland were selected by the user. The image was then transformed to polar coordinates with the center of the probe as the coordinate center. This aided in untapering and warping of the image. After initial fitting, a deformation model was used to get the final fitting of the prostate boundary traced by interacting multiple modes PDAF [25]. The obtained ellipse was used to initialize other slices of the ellipsoid. The process continued for all the slices to obtain a segmented prostate in $3 \mathrm{D}$. 


\subsection{Region based segmentation}

Predominant intensity distributions of the prostate region in different imaging modalities have been exploited by researchers to develop region based segmentation algorithms. Region based segmentation methods are further categorized into atlas, graph partitioning and level set methods.

\subsubsection{Atlas}

An atlas is created from a set of manual segmentations of an anatomical structures registered to a common coordinate frame. The atlas is then used as a reference to segment images of a new patient. Therefore, in atlas based segmentation, the segmentation problem is treated as a registration problem, since the segmentation is based on finding a one-to-one transformation mapping a pre-segmented atlas image to the new target image. Atlas guided segmentation is well suited for segmentation of structures that are stable over a large population, like the human brain [59].

\section{MRI}

Klein et al. [60] followed a multi-atlas approach to segment the prostate. Affine registration and subsequently a non rigid registration using cubic B-spline [61] in a multi-resolution framework was used to register the training volumes to the test volumes. Corresponding transformation was applied to the label images of the training dataset. In the next step most similar atlas scans were selected based on the measure of similarity computed ffrom normalized mutual information. To combine these atlas scans to into a single segmentation majority voting and STAPLE algorithm is used to produce the final segmentation. Recently, Dowling et al. [62] improved on the results obtained by [60] by introducing a pre-processing step of bias field correction, histogram equalization and anisotrpic diffusion smoothing. Dowling et al. then used rigid, affine and diffeomorphic demons registration to generate multiple labels of the test image. Most similar labels were identified and fused to generate the final segmentation. Langerak et al. [63] proposed a new schema for fusion of the labels in a multi atlas segmentation framework. They proposed to combine segmentation result of all the labels to produce the gold standard the target label. Each of the labeled images of each of the atlas was compared to the target label. Labels below a certain threshold was discarded and the target label is re-estimated with labels that have already been selected. The process continues in an iterative manner to provide the final estimated segmentation label.

\section{CT}

Acosta et al. [64] used affine and non-rigid demons registration to build a probabilistic atlas of the prostate, rectum, bladder and bones from the training images. Given a new test image the probabilistic atlas was registered using affine and demons registration and the labels of the atlas were transformed with the same transformation vector to segment the prostate, rectum, bladder and bones. Later, Acosta et al. [65] used a multi-atlas schema where similar atlases, were ranked and their labels fused to produce segmentation of the prostate, rectum, and bladder. 


\subsubsection{Graph partition}

In graph based segmentation methods pixels or group of pixels are considered as nodes while edges (gradients) between pixels are often considered as costs. The graph is then partitioned by minimizing a cost function and closely related pixels are grouped in different classes. Different graph partitioning algorithms like minimum spanning tree, minimum cut, and normalized cuts may be used for such purpose [66].

\section{TRUS}

Zouqi et al. [67] built a graph partition scheme to segment the prostate. The graph was built with nodes and edges. Pixels were the nodes while horizontal edges that connected these nodes represented edge discontinuity penalties. User defined pixels from the object and the background were used to build two special nodes: the source and the sink terminal. The max flow algorithm [68] gradually increased the flow sent from the source to the sink along the edges in the graph given their costs. Upon termination, the maximum flow saturated the graph. The saturated edges corresponded to the minimum cost cut giving an optimal segmentation. The initial contour obtained after graph cut segmentation was further refined in a fuzzy inference framework that determined the membership of a pixel based on the region based statistics.

\subsubsection{Region based level sets}

In contrast with the traditional boundary based level sets, Chan and Vese [69] used region based statistics in their energy minimization criteria to propagate the level set and segment the image. The method obtained superior results in absence of strong edges and in presence of white noise since the stopping criteria was dependent on region based statistics.

\section{TRUS}

To produce a uniform region for the prostate, Fan et al. [70] set the value of a cubical voxel to 0 if the difference between the minimum and the maximum intensity values in the voxel was below 2 . The value was set to 1 if the difference was greater than 2 but less than a threshold. This fast discriminative approach was used to extract the prostate region and used in a region based level set framework to segment the prostate in three dimensions.

\subsection{Supervised and un-supervised classification based algorithms}

In pattern recognition feature could be defined as a measurable quantity that could be used to distinguish two or more regions. More than one feature could be used to differentiate different regions and an array of these features is known as a feature vector. The vector space associated with feature vectors is known as feature space. Supervised and un-supervised classification (PR) based techniques aim at obtaining a partition of the feature space into a set of labels for different regions. Primarily classifier and/or clustering based techniques are used for the purpose. Classifiers use a set of training data with labeled objects as a priori information to build a predictor to assign label to future un-labeled observations. In contrast, in clustering methods a set of feature vectors are given and the goal is to identify groups 
or clusters of similar objects on the basis of the feature vector associated with each. Proximity measures are used to group data into clusters of similar types.

\subsubsection{Classifier based segmentation}

In classifiers based segmentation the prostate is seen as a prediction or learning problem. Each object in a training set is associated with a response variable (class label) and a feature vector. The training set is used to build a predictor that can assign class label to a object on the basis of the observed feature vector.

\section{TRUS}

Intensity heterogeneity, unreliable texture features and imaging artifacts pose challenges in the feature space to partition. Zaim [71] used texture features, spatial information and gray-level values in a self organizing map neural network to segment the prostate. In a more recent work [72] the authors used entropy and energy of symmetric, orthonormal, and second order wavelet coefficients [73] of overlapping windows in a support vector machine (SVM) classifier. Mohammed et al. [74] used spatial and frequency domain information from multi-resolution Gabor filters and prior knowledge of prostate location in TRUS images to identify the prostate. Parametric and non parametric estimation of power spectrum density of the Fourier transform along with ring and wedge filter [75] of the region of interest (ROI) were used as feature vectors to classify TRUS images into prostate and non prostate region using non linear SVM.

\subsubsection{Clustering based segmentation}

The goal of clustering based methods is to determine intrinsic grouping in a set of un-labeled data based on some distance measures. Each data is associated with a feature vector and the task is to identify groups or clusters of similar objects on the basis of the set of feature vectors. The number of groups is assumed to be known and implicitly one must select the relevant feature, distance measure and the algorithm to be used.

\section{TRUS}

Richard et al. [76] used the mean shift algorithm [77] in texture space to determine the mean and covariance matrix for each cluster. A probabilistic label was assigned to each pixel determining the membership of a pixel with respect to every cluster. Finally, a compatibility coefficient and pixel spatial information was used for probabilistic relaxation and refinement of the prostate region.

\subsection{Hybrid segmentation}

Combining a priori boundary, shape, region and feature information of the prostate gland improves segmentation accuracy. Such methods are robust to noise and produce superior results in presence of shape and texture variations of the prostate. This section discusses the methods that have combined two or more of the methods presented in previous sections. 


\section{TRUS}

Mid gland image of the prostate in axial slices in TRUS images is often characterized by a hypoechoic mass surrounded by a hyperechoic halo. In order to capture this feature, Liu et al. [78] proposed to use radial search from the center of the prostate to determine the edge points of the prostate. The key boundary point was identified from the largest variation in gray value in each line. An average shape model constructed from manually segmented contours was used to refine the key points. A similar schema was adopted by Yan et al [79]. In this case, contrast variations in normal vector profiles perpendicular to the PDM were used to automatically determine salient points and produce prostate boundaries. Salient points were determined by discarding points that fall in shadow regions. Prior shape information of the prostate shape aided determining the missing points in shadow regions in TRUS images. Optimal search performed through vector profiles perpendicular to the salient points was used to determine prostate boundary with a discrete deformable model in a multi-resolution, energy minimization framework.

Modeling shape and texture features and using them to segment a new image has been used by many researchers. The schema primarily varied in the approach adopted for the creation of the shape and the texture model. For instance, Zhan et al. [80] proposed to model the texture space by classifying into prostate and non prostate regions the texture features captured by rotational invariant Gabor filter by means of a SVM. This classified feature space was subsequently used as an external force in a deformable model framework to segment the prostate. In their consequent work [81], the authors proposed to speed-up the process by using Zernike moments [82] to detect edges in low and middle resolutions and maintaining the texture classification using Gabor features and SVM. In a different way [83], the authors also proposed to reduce the number of support vectors by introducing a penalty term in the objective function of the SVM, which penalizes and rejects the outliers. Finally, Zhan et al. [9] proposed to combine texture and edge information to improve the segmentation accuracy. Multi-resolution rotational invariant Gabor features of the prostate and non-prostate regions were used to train a Gaussian kernel SVM system to classify textures of prostate regions. In the deformable segmentation procedure, SVM are used to label voxels around the surface of deformable model as prostate or non prostate tissues. Subsequently, the surface of deformable model is driven to the boundary by the deformation force of labeled prostate tissues. The step of tissue labeling and the step of label-based surface deformation being dependent on each other, the process is carried out iteratively to convergence.

A similar schema was adopted by Diaz and Castaneda [84]. Asymmetric stick and anisotropic filters were firstly applied to reduce speckle in TRUS images. A DDC was produced using cubic interpolation of four points initialized by the user. The DDC deformed under the influence of internal force, gradient magnitude and damping forces to produce the contour of the prostate. Features such as intensity mean, variance, output of back projection filter, and stick filter were used to construct the feature vectors. The pixels were classified into prostate and non prostate regions using SVM. Subsequently, DDC was automatically initialized from the prostate boundary and used to obtain the final contour of the prostate. Cosío et al. [10] used position and gray scale value of a prostate in TRUS image in a Gaussian mixture model of three Gaussian to cluster prostate, and non prostate tissues and to identify halo around the prostate in TRUS images. Bayes classifier was used to identify prostate region. After pixel classification the ASM is initialized 
with the binary image using a global optimization method. The optimization problem consists of finding the optimum combination of four pose and two shape parameters, which correspond to an approximate prostate boundary in the binary image. A multi population genetic algorithm with four pose and ten shape parameters was used to optimize an ASM in a multi-resolution framework to segment the prostate.

Another common hybrid approach is to use both shape and intensity distribution to segment the prostate. Medina et al. [85] used an AAM framework [86] to model the shape and the texture space of the prostate. In this framework the Gaussian model of the shape and intensity created from PCA analysis is combined to produce a combined mean model. The prostate was segmented exploiting the prior knowledge of the nature of the optimization space in minimizing the difference between the target image and the mean model. Ghose et al. [87] used the approximation coefficients of Haar wavelets to reduce speckle to improve on segmentation accuracies. Later, Ghose et al. [88] improved the model further by introducing contrast invariant texture features extracted from log Gabor quadrature filters. More recently Ghose et al. [89] used probabilistic information obtained in a Bayesian framework to build the appearance model. Furthermore, multiple mean models of shape and appearance priors were used to improve on segmentation accuracies. Gong et al. [90] proposed to use a deformable super ellipse to produce a shape model of the prostate. Using the deformable super ellipse as the prior shape model for the prostate, the end goal was to find the optimal parameter vector that best describes the prostate in a given unsegmented image. The search was formulated as a maximum a posterior criterion using the Bayes rule. The initial parameters were used in maximum a posteriori (MAP) framework to obtain the optimized parameters for the ellipse.

Later, Tutar et al. [91] used the average of three manually delineated prostate contours to construct a three dimensional mesh with spherical harmonics to represent the average model of the prostate. With 8 harmonics, a feature vector of 192 element was reduced to 20 using PCA. Users initialize the algorithm by outlining the prostate boundaries in mid gland axial and sagittal images. Therefore, the problem of finding the shape parameter vector that would segment the prostate in spatial domain was reduced to find the optimal shape parameters in parametric domain that maximized the posterior probability density of a cost function, which measures the degree of agreement between the model and the prostate edge in the image. Yang et al. [92] proposed to use min/max flow [93] to smooth the contours of the 3D model of the prostate created from 2D manual delineation. The primary modes of shape variations were identified with PCA and morphological filters were used to extract region based information of the prostate gland. The shape model and region based information were then combined in a Bayesian framework to produce an energy function, which was minimized in a level set framework. Garnier et al. [94] used 8 user defined points to initialize a 3D mesh of the prostate. Two algorithms were used to determine the final segmentation of the prostate. First, DDC with edge as external force and the 6 central gland user defined points as landmarks was used to deform the mesh to segment the prostate. Next, the initial mesh was used to create the graph and in second stage image features like gradients were introduced to build the cost function. Finally, graph-cut was used to determine the prostate volume. The graph cut results were refined with DDC to improve the results. 


\section{MRI}

Prior shape and size information of the prostate were exploited by Vikal et al. [95] to build an average shape model from manually delineated contours. The authors used the Canny filter to determine edges after pre-processing the images with a stick filter to suppress noise and enhance the contrast. The average shape model was used to discard pixels that did not follow similar orientation as the model. The obtained contour was further refined by the removal of gaps using polynomial interpolation. The segmented contours obtained in the middle slices were used to initialize slices lying above and below the central slice.

The use of a Bayesian framework to model the texture of the prostate is common in MR images. For instance, Allen et al. [96] proposed to segment the prostate in an EM framework treating the three distinctive peaks in intensity distribution as mixture of three Gaussians (background, central region and periphery of the prostate). A shape restricted deformable model with the clustered pixels as a deformation force was then used to segment the prostate. Similarly, in Makni et al. [3], the intensities of the prostate region were modeled as a mixture of Gaussians. They proposed a Bayesian approach where the prior probability labeling of the voxels was achieved by using a shape restricted deformable model and Markov field modeling. The conditional probability was associated with the modeled intensity values, and the segmentation was achieved by estimation of an optimum label for prostate boundary pixels in a MAP decision framework.

Although atlas based registration and segmentation of the prostate has become popular in recent time, the obtained segmentation results had to be refined with a deformable model to improve the accuracy. Martin et al. [2] used a hybrid registration minimizing intensity and geometry energies for registering the atlas. The minimization of the intensity based energy aimed at matching the template image with the reference image while the minimization of the geometric energy matched the model points of the template image to the scene points belonging to the reference image. Finally, a shape constrained deformable model was used to refine the results. More recently, Martin et al. [97] used a probabilistic atlas to impose further spatial constraints and segment the prostate in three dimensions.

Shape and texture modeling of the prostate were merged in the work of Tsai et al. [98], who used a shape and region based level set framework to segment prostate in MR images. One of the contours was fixed and used as the reference system where all the other contours were affine transformed to minimize their difference in a multi resolution approach. PCA of the shape variability captured the primary modes of variations and was also incorporated in the level set function, along with region based information such as area, sum of intensities, average intensity and variance information. The minimization of the level set objective function produced the segmented prostate. The authors also suggested a coupled level set model of the prostate, the rectum, and the internal obturator muscles from MR images to segment these structures simultaneously [99]. The algorithm was made robust by allowing the shapes to overlap with each other, and the final segmentation was achieved by maximizing the mutual information of the three regions. Similarly, Liu et al. [6] used a deformable ellipse to segment prostate boundary after Otsu thresholding [100] of the image in prostate and non prostate region. A shape constrained level set initialized from the elliptical fitting of the prostate was used to further refine the results. Finally, post processing of the gradient map of the prostate 
and the rectum produced the final segmentation. Firjani et al. [101] modeled the background and the foreground pixels with Gaussian mixture Markov random field and used the information of probability of a pixel being prostate in building the shape model. The shape and the intensity were jointly optimized with a graph cut based algorithm. The authors extended their work for a 3D segmentation of the prostate [102]. Zhang et al. [103] proposed an interactive environment for prostate segmentation. Region and edge based level sets were used to segment the prostate from the background depending on foreground and background region based information provided by the user.

Gao et al. [104] represented the shapes of a training set as point clouds. Particle filters were used to register clouds of points created from prostate volumes to a common reference to minimize the difference in pose. Shape priors and local image statistics were incorporated in an energy function that was minimized to achieve prostate segmentation in a level set framework. More recently, Toth et al. [105] used a series of 50 Gaussian kernel of variable size to extract prostate texture features. ASM constructed from manually delineated contours of training images was automatically initialized depending on the most probable location of the prostate boundary to achieve segmentation. Later, Toth et al. [106] in addition to intensity values, used mean, standard deviation, range, skewness, and kurtosis of intensity values in a local neighborhood to propagate ASM automatically initialized from magnetic resonance spectroscopy (MRS) information. MRS information was clustered using replicated k-means clustering to identify prostate in mid slice to initialize multi feature ASM. Khurd et al. [107] localized the center of the prostate gland with Gaussian mixture model and expectation maximization based clustering after reducing magnetic bias in the images. Thresholding on the probabilistic map of the prostate obtained with random walker based segmentation algorithm [108] to segment the prostate.

\section{CT}

As shown in Figure 1(c), the prostate gland in $\mathrm{CT}$ images shows an uniform intensity distribution and poor contrast between the gland shape and its surrounding tissues. Hence, combining shape information with region based statistics is a common approach when segmenting CT images, since the prior shape information restricts the deformation to viable shapes while the region based statistics propagate the deformation. For instance, Freedman et al. [109] used manually delineated contours to form a three dimensional mesh in which the contours were interpolated using splines. The intensity information of each slice was incorporated in a probability density function (PDF) framework. For segmentation, each slice was intersected with the mesh yielding a series of polygons and their corresponding histograms, which were added and normalized to get the distribution of each of the slices. The segmentation was finally achieved by the minimization of the cumulative distribution function of the PDF between the model and the image slices. Similarly, Rousson et al. [110] used two shape constrained level sets for simultaneously segmenting the prostate and the bladder. A non overlapping constraint was imposed to drive the prostate and bladder apart by assuming that the two level sets evolved independent from each other and a penalty term was introduced whenever the two voxels were shared. Davis et al. [111] proposed to quantify organ motion for adaptive radiation therapy using deformable registration. Bowel gas was segmented using thresholding and morphological filtering and then using gradient direction to collapse the bowel. Finally, deformable registration was used to segment the prostate. In 
contrast, Ghosh et al. [112] used a genetic algorithm to guide the level set that segmented the prostate. The pose and weight parameters of manually delineated contours were used as inputs to the genetic algorithm, and the fitness function was constructed based on texture features. The pixels inside the generated curve were grouped depending on Laws texture values and the fitness score was generated. This score was maximum when all the landmarks were in a texture similar to the one of the prostate region. Subsequently, the curves were ranked according to the fitness scores and the higher ranking curves were chosen to produce children by mutation and crossover. The process continued for several iterations until convergence. Similarly, Costa et al. [113] used PCA to impose shape constraints and used region based statistics in an energy minimization framework. A local affine registration of the pelvic bones was used to localize the prostate and the bladder from prior spatial information. A morphological filtering followed by a modified region growing was used to obtain an initial estimate of the segmentation of the bladder. A non overlapping constraint created from the distance potential of the prostate and bladder was used to drive the two structures apart during segmentation.

Finally, shape, edge and region based statistics can be also combined for segmenting the prostate. Song et al. [114] used an arc weighted graph [115] for incorporating shape constraints and edge information for segmentation of the bladder and the prostate. Prior shape knowledge was introduced using the weights of both graph nodes and directed graph edges or arcs. A three dimensional level sets were used for a rough segmentation of the bladder and manually delineated contours of the prostate were used to construct the mean shape and fit it to a CT image using rigid transformation. Recently, Song et al. [116] improved the segmentation accuracy by incorporating region based intensity distribution information and using both boundary and region based energy in an energy minimization framework optimized with graph cut. Chen et al. [117] used three level sets for the prostate, bladder and the rectum that deformed under the influence of distance signed function computed from shape statistics, gradients, PDF of the region and a smoothness constraint to segment the three organs. Chen and Radhke [118] also used shape and intensity priors in their level set framework. Kernel density estimation was used for both shape and intensity priors to construct an energy function that was minimized using gradient descent optimization. Feng et al. [119] used profile-based gradient features and the local-region based probability distribution function to build appearance model. ASM used the appearance model for deformation to segment the prostate. Gradient and probability distribution function combined feature produces more accurate and robust segmentations than general gradient features for ASM. An on line learning mechanism was used to build shape and appearance statistics for accurately capturing intra-patient variation.

$\mathrm{Li}$ et al. [120] used rigid alignment of the pelvic bone structures to align the training images. Features like appearance, context, Haar like features, histogram of oriented gradients and pixel coordinates were extracted in 2D inside a region of interest to train two location adaptive classifier. Given a new image the two classifiers were used to produce two probabilistic map of the location of the prostate. The two maps were fused and a level set is used to produce a binary classification. Finally, absolute intensity variation between the context location (obtained from the map) and current pixel was used in the classifier to update the classifier and produce final segmentation. Liao et al.[121] uses rigid alignment of the bone structures to localize prostate. Localized multiresolution Haar wavelets, 
histogram of oriented gradients, and local binary patterns features were extracted and salient features were selected from a Gaussian function developed from Dice ratio. An online learning was used to integrate both inter and intra patient specific information to localize prostate from a sigmoid function. Given a new image learn't image similarity function was utilized to align new patient for segmentation with a support vector regression. Lu et al. [122] shape and region prior level sets to segment the prostate and a B spline based registration was used to minimize the cross correlation value between the image in training stage and treatment stage to constrain the segmentation results. The segmentation and registration framework worked iteratively to produce the final segmentation. Chen et al. [123] used median and morphological filtering on k means clustering to identify bones, muscles and gas in images. Slice by slice segmentation of the bones were performed to identify pelvic and coccyx bones to impose anatomical constraint in prostate segmentation. PCA of anatomical landmarks generated using Fourier descriptors weer used to identify shape variations. Finally, registration on intensity in region of interest was obtained. Shape, anatomical and registration were all used in a Bayesian framework to achieve segmentation.

\section{Validation and qualitative performance evaluation}

The performance of prostate segmentation algorithms is usually evaluated comparing the output of the method with a ground truth (gold standard) obtained from manual delineations of the prostate done by experienced radiologists. Hodge et al. [46] advised to use the mean of the manual segmentations of different radiologists and/or of the same radiologist at different times to reduce inter and intra observer variations in preparation of the ground truth value.

Analyzing the literature we have seen that the evaluation metrics could be categorized into qualitative and quantitative based metrics. In a qualitative evaluation, the obtained contour is visually compared with the ground truth value. In contrast, for quantitative evaluation, an error between the obtained contour and the ground truth is numerically computed. Typically, these error metrics could be classified into contour based, area based and volume based methods. Contour based metrics rely on computing how close the ground truth and the obtained contours are. Typical metrics used are the Hausdorff distance (HD) [124], the mean absolute distance (MAD) [79], mean distance (MD) [27], maximum distance (MaxD) [78], and root mean square error (RMSQ) [47]. Area based errors are based on computing how much the ground truth and the obtained area overlap. It can be measured by the Dice similarity coefficient (DSC) [125], area accuracy [56], area sensitivity [56], area specificity [84], area overlap [25], area overlap error [44], and area error [27] metrics. Finally, volume overlap error and difference, average difference [33], overlap [91], detection, false detection, centroid distance [109], and similarity [123] are used for computing a 3D overlapping error. However, DSC, specificity, sensitivity, accuracy and HD of voxels are also used in terms of voxels to determine volumetric overlap [104]. The evaluation metrics for prostate segmentation is enlisted in Table 3 and 4.

Ideally a comparison of different state-of-the-art prostate segmentation methodologies on a public dataset should have been done to evaluate the performance of the state-of-the-art methods. However, a quantitative comparison of different methodologies is difficult in absence of public software, data sets and standardized evaluation metrics. In 
addition, the methods are developed using wide variety of algorithms with specific application requirements. Hence, such a quantitative comparison of different prostate segmentation methods on the same dataset with some standardized metrics is extremely difficult as could be observed in some recently published works [2, 10, 44, 58, 79, 90, 97, 104]. Nevertheless, to have an overall quantitative estimate of the functioning of some of the state-of-the-art works in the literature we present the reported results in Tables 5, 6, 7 and 8 for TRUS, MRI and CT imaging, respectively.

The index of the Tables is expanded below.

- The name of the first author has been used as a reference of the paper.

- The segmentation dimension (Dim) gives the output of a given segmentation methodology. The output can be in two (2D) or three (3D) dimensions.

- B/A indicates whether base and apex slices were considered for 2D segmentation.

- Pre-Proc indicates the type of pre-processing used in the method.

- In. indicates the use of endo-rectal coil in acquisition of MR images.

- The segmentation criteria shows in what of the reviewed categories should be classified the analyzing segmentation algorithm. Hybrid segmentation methodologies are specified with the type of algorithms that are combined to produce the final segmentation (the acronyms of this row are: DM $=$ Deformable model, $\mathrm{ASM}=$ Active shape model, $\mathrm{AAM}=$ Active appearance model, $\mathrm{GA}=$ Genetic algorithm, $\mathrm{EM}=$ Expectation maximization, DDC $=$ Discrete dynamic contour, $\mathrm{ACM}=$ Active contour model, $\mathrm{SVM}=$ Support vector machine, $\mathrm{ANN}=$ Artificial neural network, S-R Level set $=$ Shape and region based level set).

- The automation (Auto) column specifies the degree of manual interaction that was necessary. The process is considered automatic if the degree of manual interaction was restricted to training.

- The measure column refers to the measures used by the authors to present their obtained results.

- The last column (Validation) gives the number of images or data sets (volumes) that were used to validate the developed algorithm.

The tables are firstly analyzed according to the imaging modality. Afterwards, a brief discussion on the evaluation procedures is given in section 4 .

\subsection{Open problems}

We have explained in previous sections the validation procedures followed by the researchers. From the reported results a set of of open problems are revealed. 
1. Manual delineation of the prostate contours is considered to be the gold standard to which the result of a segmentation methodology is compared. Only few authors considered the mean of delineated contour by different experts and of the same expert at different time to reduce inter and intra observer variability of the process. Rasch et al. [126] quantified inter observer variabilities of CT and MRI. He found that, the average ratio between the volume derived by one observer for a particular scan and patient and the average volume was 0.95 , 0.97, and 1.08 for the three observers. Under such inter observer variabilities an interesting option could be the use of prostate phantom to validate volume information obtained using computer aided segmentation.

2. The unavailability of public prostate database makes quantitative comparison of the segmentation algorithms difficult. Moreover, the quality of results depend on the both the scans and quality of contouring. Lately, MICCAI prostate challenge datasets for MRI are being used for comparison [104]. A public datasets of prostate images [127] could also be used for validation. The quality of the images vary with CT, MR and TRUS machines, as advanced machines produce images of superior quality. Thus, it becomes almost impossible to compare the performance of two algorithms separated by a span of significant number of years.

3. Lack of standardized metrics in evaluation of segmentation result makes the comparison of developed methodologies difficult as shown in Table 3 and Table 4. Mean average distance, maximum distance, average distance, area of overlap, area difference, volume overlap and volumetric error are just a few of the commonly used metrics. However, since MICCAI prostate challenge 2009 [125], Hausdorff distance and DSC are being increasingly used.

4. Very few fully automatic methods have been developed, and often manual initialization and sometimes manual editing is encouraged. This may be suitable for off-line procedures like the estimation of prostate volume, but unsuitable for on-line procedures like real time fusion of multi-modal images [128].

\section{Choosing an appropriate segmentation method}

Choice of a proper segmentation methodology is dependent on contexts like imaging modality, and the final target application of the process. Hence, we have provided recommendation of selection of a particular segmentation technique based on these two basis. We have divide the section into TRUS, MRI and CT subsections and have provided recommendations based on applications in each of these modalities.

\subsection{TRUS}

TRUS image of a prostate has low contrast and the signal is often corrupted by speckle, shadow artifacts and micro-calcifications [79]. There are two different ways to deal with speckle. One option is to minimize its effect in the image using, for example, stick filters [17], that allow reducing speckle while enhancing the contrast of the image. The second option is to take benefit of this information, which can be done modeling speckle as a Rayleigh's distribution [57]. Any of these options could be employed for pre-processing of the image and prepare it for further analysis. 
Prostate volume determined from segmented TRUS images serves as an important parameter in determining presence of benign or malignant tumor during diagnosis of prostate diseases. Three commonly used prostate volume measurement techniques in TRUS are planimetry calculation, prolate ellipse volume calculation, and an ellipsoid volume measurement technique. Segmentation of prostate in 2D in the axial slices in the mid gland region to determine maximum area and height is useful in determining volume in all these techniques. Note in Table 5 that no one tried a pure region based approach to segment the prostate. This is due to the fact that these algorithms fragment the prostate into a large number of small regions due to the heterogeneity inside the prostate gland. Related with edge based approaches, we noted that pure contour based methods like edge detection [17] are being replaced (or expanded) with methods that combine prostate shape and region based information [9, 10, 84], providing a more robust approach in presence of speckle and low contrast. In contrast, Abolmaesumi et al. [25] and Sahba et al. [27] proposed an interesting option of modeling the prostate contour as a Gaussian distribution. Such assumption provides more robustness to contour based methods in low contrast. ASM is another edge based approach frequently used for prostate segmentation in 2D images. However, such models are dependent on reliable edge information and hence may be adversely affected in presence of shadow artifacts [79]. Moreover, the automatic initialization and extension to 3D is difficult [47]. However, shape constrained deformable models have been successfully employed by different authors $[9,91,92]$ as observed in Table 5. Automatic delineation of the prostate in mid-gland images further reduces inter observer variabilities. The method of Ghose et al. [89] could be used for the purpose it is automatic, fast and accurate.

In prostate brachytherapies, oncologists should prepare a set of parallel TRUS ultrasound images and manually segment each 2D slice to obtain the prostate volume which is then used to plan the location of the seeds. Hence, fast seimi-automatic or automatic prostate segmentation in 2D slices or 3D volume could be useful in such procedures. Mahdavi et al. [58] method of fitting an ellipsoid to prostate edges is a very useful method for such a scenario as the method has shown good volumetric overlap accuracy.

Automatic, and fast prostate segmentation from 2D US images is often necessary in image guided prostate biopsy or robot assisted surgery [79]. DDC and super quadrics are computationally efficient procedures to segment the prostate in 2D. However, the fastest segmentation of the prostate contour had been reported using partial ASM [79] and probabilistic filtering [25]. Considering semi-automatic approach adopted by [25], the method developed by Yan et al. [79] is well suited for real time segmentation of the prostate in two dimensions. Note that the speed of a given segmentation method could be improved if the method could be parallelized and implemented in graphical processing unit as well as if an off-line learning of the optimization space could be adopted as proposed by Ghose et al [88]. Moreover, segmentation of prostate in TRUS videos could be modeled as a tracking boundary problem to achieve near real time segmentation.

Supervised and un-supervised classification based methods have the advantage of being fully automatic [71, 74], although a training is necessary in the ones using a classifier. However, the intensity heterogeneity and unreliable texture of the prostate gland challenge again the development of a pure clustering or classification schema for the 
prostate. Therefore, an interesting and common option is the use of a clustering or/and classification schema for initial segmentation and subsequently use the obtained information for the initialization and/or propagation of a deformable model to produce the final segmentation [9]. Another common hybrid approach is the integration of shape and intensity information jointly optimized in an AAM framework [85]. Off-line learning of the optimization space aids in fast prostate segmentation. However, such methods are affected by large-scale contrast variances and use of texture information in place of raw intensity improve segmentation accuracies [88].

\section{2. $M R I$}

MR images of the prostate have better soft tissue contrast compared to US or CT images. However, insertion of the endorectal coil to enhance contrast in the prostate region introduces intensity inhomogeneities that may adversely affect the segmentation accuracy of algorithms dependent on pixel intensities. Viswanath et al. [129] performed an extended review of three techniques [130], [131] and [132] applied to magnetic field bias field correction. The authors concluded that bias field correction algorithm should be application specific. For example it was observed that [130] performed best with the goal of identifying cancer and on the other hand bias field corrections can adversely affect clustering and classification based techniques of segmentation. It is to be noted that MR images with endorectal coil are relatively simpler to segment due higher contrast of images around the prostate and well deformed shape of the rectum.

Prostate segmentation from MR images are frequently used for volume determination, surgical planing and multimodal image registration. In all these applications prostate segmentation could be done automatically or semiautomatically. However, minimum human interaction is desired to minimize human induced variations and errors. In the last decade, deterministic and probabilistic atlases are frequently used for 3D segmentation of the prostate. Such methods are automatic, robust to intensity variability and to noise [60]. Martin et al. used both deterministic atlas [2] and probabilistic atlas [97] to segment the prostate, although the obtained segmentations were refined with a deformable model. Pair wise atlas selection schema of Dowling et al. [62] has shown greater accuracy compared to $[60,133]$. Hence, for atlases pairwise registration is better compared to average atlas based segmentation. In Table 7 we observe that Klein et al. [60] with atlas based segmentation achieved an impressive overlap accuracy of 0.85 DSC value when validated with 50 data sets. However, Martin et al. [97] with probabilistic atlas and deformable model based segmentation achieves similar overlap accuracy and a good contour accuracy values when validated with 36 data sets. Atlas based methods [60] and probabilistic modeling of the prostate region [2] provide a more robust approach in presence of these inhomogeneities.

Deformable models are frequently used for prostate segmentation. Makni et al. [3] used information coming from an initial classification scheme to initialize a deformable model. Note also that automatic methods are primarily developed using classifiers, atlas and deformable models. It has to be noted that anatomical structures around the prostate may affect the prostate deformation. Modeling the anatomical structures like bladder and rectum along with prostate will provide additional flexibility to the segmentation algorithm [99]. A hybrid segmentation method that 
incorporates shape and intensity priors achieves good segmentation accuracy $[98,104]$. Accuracies of segmentation of prostate in MRI using 3D ASM depends on initialization. Cosio et al. [10] provided an efficient initialization scheme in their work using Bayesian classification. In recent years Toth et al. [106] have used clustering of spectral data obtained in DCE MRI to initialize 3D ASM. Segmentation accuracies were improved using feature driven ASM.

\section{3. $C T$}

Prostate segmentation from CT images is extremely important for patient undergoing radiation therapy. In such cases automatic segmentation of intra-treatment $\mathrm{CT}$ images for adaptive radiation therapy of the prostate is useful. In adaptive radiation therapy periodic inter-treatment $\mathrm{CT}$ images are used for localization of the tumor and a feedback control strategy is used to correct the differences between planned and delivered dose distribution due to spatial changes in the treatment volume [111]. Also, in radiation therapy it is essential to ensure accurate delivery of the target dose under organ motion. Often, gold fiducial markers in or adjacent to the target in image guided radiotherapy is used to correct day-to-day variations in the target position as these fiducial markers being radio-opaque could be used to as a visible surrogate [134]. The fiducial markers are frequently used for motion estimation and to accurately locate region of interest [135] and motion correction is useful for segmentation of prostate in inter-treatment prostate images [111]. Segmentation of prostate in intra-treatment CT images is important for adaptive treatment planing and often same patient image is used to model and segment the prostate. Tang et al. [48], Feng et al. [49], Freedman et al. [109], [111], and Song et al. [114] have all used same patient for training and segmentation.

Poor contrast between prostate and surrounding tissues makes the prostate segmentation difficult in CT images. We observe in Table 8 that intensity homogeneity of the prostate region in CT images has been frequently used for designing models that exploit shape and region information. Poor tissue contrast between the prostate gland and the surrounding tissues inhibits methods that work on boundary information, and hence, shape prior information constraints the propagation of deformable models in absence of strong edges. Building shape restricted level sets propagating on intensity statistics is well suited for prostate segmentation $[109,110,113,117,122]$.

\section{Conclusion and future trends}

Diagnostic imaging has become an indispensable procedure in medical science. Methods of imaging the patient anatomical structures have improved the diagnosis of pathologies, creating new avenues of research in the process. Automatic segmentation of anatomical structures from different imaging modalities like US, MRI and CT has become an essential step to reduce inter and intra-observer variability, improving contouring time thereafter. This paper reviewed the methods involved with prostate segmentation. Strength and limitations of the segmentation methodologies have been discussed along with the presentation of validation and performance evaluations of the same. Finally, a discussion on choosing an appropriate segmentation methodology for a given imaging modality has been carried out. It has been highlighted that prostate segmentation techniques should utilize geometric, spatial, intensity, texture, and imaging physics priors to improve accuracy. 
Prostate segmentation is still an open problem and with advancement of technology for diagnosis, treatment and follow up of prostate diseases new requirements have to be met. Multimodal image fusion of at least two imaging modalities provides valuable information. For example, the fusion of MRI and TRUS imaging should aid in obtaining more accurate samples during biopsies. However, for such a method to work in a real scenario, automatic, accurate and real time fusion of the two imaging modalities is necessary. Under such circumstances automatic real time segmentation of the prostate and registration on prostate contours would improve accuracy and efficiency. Automatic and accurate real time segmentation of the prostate may be achieved with efficient algorithms designed for graphical processing units. Moreover, the goal of segmenting the prostate in every frame could be modified with the objective of tracking prostate in every frame. An increase in 3D prostate segmentation methods will be the trend in coming years due to the increasing use of 3D imaging modalities, where efficient and accurate algorithms are necessary. In that sense, information from dynamic contrast enhanced MRI, and MR spectroscopy will be increasingly used as additional features for automatic segmentation. In addition, registration done on prostate contour for the same modality over a period of time may provide also valuable information about the progression of a prostate disease.

\section{Conflict of interest}

All authors in this paper have no potential conflict of interests.

\section{Acknowledgements}

This research has been funded by VALTEC 08-1-0039 of Generalitat de Catalunya, Spain and Conseil Régional de Bourgogne, France. The research is partially funded by Spanish science and innovation grant nb.TIN2011-23704.

[1] Prostate Cancer Statistics - Key Facts, info.cancerresearchuk.org/cancerstats/types/prostate, accessed on [1st April, 2011], 2011.

[2] S. Martin, V. Daanen, J. Troccaz, Atlas-based Prostate Segmentation Using an Hybrid Registration, International Journal of Computer Assisted Radiology and Surgery 3 (2008) 485-92.

[3] N. Makni, P. Puech, R. Lopes, R. Viard, O. Colot, N. Betrouni, Combining a Deformable Model and a Probabilistic Framework for an Automatic 3D Segmentation of Prostate on MRI, International Journal of Computer Assisted Radiology and Surgery 4 (2009) 181-8.

[4] S. Ozer, D. L. Langer, X. Liu, M. A. Haider, T. H. van der Kwast, A. J. Evans, Y. Yang, M. N. Wernick, I. S. Yetik, Supervised and Unsupervised Methods for Prostate Cancer Segmentation with Multispectral MRI, Medical Physics 37 (2010) $1873-83$.

[5] P. Puech, N. Betrouni, N. Makni, A.-S. Dewalle, A. Villers, L. Lemaitre, Computer-Assisted Diagnosis of Prostate Cancer Using DCE-MRI Data: Design, Implementation and Preliminary Results, International Journal of Computer Assisted Radiology and Surgery 4 (2009) 1-10.

[6] X. Liu, D. L. Langer, M. A. Haider, Y. Yang, M. N. Wernick, I. S. Yetik, Prostate Cancer Segmentation with Simultaneous Estimation of Markov Random Field Parameters and Class, IEEE Transactions on Medical Imaging 28 (2009) 906-15.

[7] E. J. Halpern, D. L. Cochlin, B. B. Goldberg, Imaging of the prostate, Informa Healthcare, United Kingdom, first edition, 2002.

[8] F. Shao, K. V. Ling, W. S. Ng, R. Y. Wu, Prostate Boundary Detection From Ultrasonographic Images, Ultrasound in Medicine 22 (2003) $605-23$.

[9] Y. Zhan, D. Shen, Deformable Segmentation of 3D Ultrasound Prostate Images Using Statistical Texture Matching Method, IEEE Transactions on Medical Imaging 25 (2006) 256-72.

[10] F. A. Cosío, Automatic Initialization of an Active Shape Model of the Prostate, Medical Image Analysis 12 (2008) 469-83.

[11] Y. Zhu, S. Williams, R. Zwiggelaar, Computer Technology in Detection and Staging of Prostate Carcinoma: A Review, Medical Image Analysis 10 (2006) 178-99. 
[12] J. A. Noble, D. Boukerroui, Ultrasound Image Segmentation: A Survey, IEEE Transactions on Medical Imaging 25 (2006) 987-1010.

[13] D. L. Pham, C. Xu, J. L. Prince, Current Methods in Medical Image Segmentation, Annual Review of Biomedical Engineering 2 (2000) $315-7$.

[14] Y. J. Liu, W. S. Ng, M. Y. Teo, H. C. Lim, Computerised Prostate Boundary Estimation of Ultrasound Images Using Radial Bas-Relief Method, Medical and Biology Engineering and Computing 35 (1997) 445-54.

[15] C. K. Kwoh, M. Y. Teo, W. S. Ng, S. N. Tan, L. M. Jones, Outlining the Prostate Boundary Using the Harmonics Method, Medical and Biology Engineering and Computing 36 (1998) 768-71.

[16] R. G. Aarnink, S. D. Pathak, J. J. M. C. H. de la Rosette, F. M. J. Debruyne, Y. Kim, H. Wijkstra, Edge Detection in Prostatic Ultrasound Images Using Integrated Edge Map, Ultrasonics (1998) 635-42.

[17] S. D. Pathak, V. Chalana, D. R. Haynor, Y. Kim, Edge-Guided Boundary Delineation in Prostate Ultrasound Images, IEEE Transactions on Medical Imaging 19 (2000) 1211-9.

[18] R. Zwiggelaar, Y. Zhu, S. Williams, Semi-Automatic Segmentation of the Prostate, in: F. J. Perales, A. J. Campilho, N. P. de la Blanca, A. Sanfeliu (Eds.), Pattern Recognition and Image Analysis, Proceedings of First Iberian Conference, IbPRIA, Springer, Berlin and Heidelberg and New York and Hong Kong and London and Milan and Paris and Tokyo, 2003, pp. 1108-16.

[19] T. Lindeberg, Edge Detection and Ridge Detection With Automatic Scale Selection, in: Proceedings of Computer Vision and Pattern Recognition, IEEE Computer Society Press, Los Alamitos, California, Washington and Brussels and Tokyo, 1996 , pp. 465-70.

[20] M. Samiee, G. Thomas, R. Fazel-Rezai, Semi-Automatic Prostate Segmentation of MR Images Based on Flow Orientation, in: IEEE International Symposium on Signal Processing and Information Technology, IEEE Computer Society Press, USA, 2006, pp. $203-7$.

[21] D. Flores-Tapia, G. Thomas, N. Venugopal, B. McCurdy, S. Pistorius, Semi Automatic MRI Prostate Segmentation Based on Wavelet Multiscale Products, in: 30th Annual International Conference of the IEEE Engineering in Medicine and Biology Society, IEEE Computer Society Press, USA, 2008, pp. 3020-3.

[22] G. Welch, G. Bishop, An introduction to kalman filter, http://www.cs.unc.edu/ welch/kalman/kalmanIntro.html, accessed on [19th May, 2011], 2011.

[23] C. Rasmussen, G. D. Hager, Probabilistic Data Association Methods for Tracking Complex Visual Objects, IEEE Transactions on Pattern Analysis and Machine Intelligence 23 (2001) 560-76.

[24] P. M. Djuric, J. H. Kotecha, Z. Jianqui, H. Yufei, T. Ghirmai, M. F. Bugallo, J. Miguez, Particle Filtering, IEEE Signal Processing Magazine, 20 (2003) 19-38.

[25] P. Abolmaesumi, M. Sirouspour, Segmentation of Prostate Contours from Ultrasound Images, in: Proceedings of IEEE International Conference on Acoustics, Speech, and Signal Processing, IEEE Computer Society Press, USA, 2004, pp. 517-20.

[26] S. Z. Li, Markov Random Field Models in Computer Vision, Spinger-Verlag New York Berlin Heidelberg Tokyo, first edition, 1995.

[27] F. Sahba, H. R. Tizhoosh, M. M. A. Salama, Segmentation of Prostate Boundaries Using Regional Contrast Enhancement, in: IEEE International Conference on Image Processing, IEEE Computer Society Press, USA, 2005, pp. 1266-9.

[28] I. N. Bankman, Handbook of Medical Image Processing and Analysis, Elsevier, USA, second edition, 2008.

[29] M. Kass, A. Witkin, D. Terzopoulos, Snakes: Active Contour Models, International Journal of Computer Vision 1 (1988) 321-31.

[30] L. Cohen, I. Cohen, Finite Element Methods for Active Contour Models and Balloons for 2-D and 3-D Images, IEEE Transaction on Pattern Analysis Machine Intelligence and Image Processing 15 (1993) 1131-47.

[31] S. T. Acton, N. Ray, Biomedical Image Analysis: Tracking, Morgan and Claypool Publishers, USA, first edition, 2005.

[32] C. Xu, J. L. Prince, Snakes, Shapes, and Gradient Vector Flow, IEEE Transaction on Image Processing 7 (1998) $359-69$.

[33] C. Knoll, M. Alcañiz, C. Monserrat, V. Grau, M. C. Juan, Outlining of the Prostate Using Snakes with Shape Restrictions Based on the Wavelet Transform (doctoral thesis: Dissertation), Pattern Recognition 32 (1999) 1767-81.

[34] H. M. Ladak, F. Mao, Y. Wang, D. B. Downey, D. A. Steinman, A. Fenster, Prostate Segmentation from 2D Ultrasound Images, in: Proceedings of the 22nd Annual International Conference of the IEEE Engineering in Medicine and Biology Society, IEEE Computer Society Press, Chcago, USA, 2000, pp. 3188-91. 
[35] S. Lobregt, M. A. Viergever, A Discrete Dvnamic Contour Model, IEEE Transactions on Medical Imaging 14 (1995) $12-24$.

[36] M. Ding, C. Chen, Y. Wang, I. Gyacskov, A. Fenster, Prostate Segmentation in 3D US Images Using the Cardinal-Spline Based Discrete Dynamic Contour, in: J. Robert L. Galloway (Ed.), Proceedings of SPIE Medical Imaging : Visualization, Image-Guided Procedures, and Display, SPIE, USA, 2003, pp. 69-76.

[37] A. Jendoubi, J. Zeng, M. F. Chouikha, Segmentation of Prostate Ultrasound Images Using an Improved Snakes Model, in: Proceedings of 7th International Conference on Signal Processing, IEEE Computer Society Press, USA, 2004, pp. $2568-71$.

[38] A. Zaim, J. Jankun, An Energy-Based Segmentation of Prostate from Ultrasound Images Using Dot-Pattern Select Cells, in: IEEE International Conference on Acoustics, Speech and Signal Processing, IEEE Computer Society Press, USA, 2007, pp. 297-300.

[39] A. Ghanei, H. Soltanian-Zadeh, A. Ratkewicz, F.-F. Yin, A Three-Dimensional Deformable Model for Segmentation of Human Prostate from Ultrasound Images, Medical Physics 28 (2001) 2147-53.

[40] P. H. Todd, R. J. Y. McLeod, Numerical Estimation of the Curvature of the Surfaces, Computer-Aided Design 18 (1986) $33-7$.

[41] K. R. Rao, J. Ben-Arie, Optimal Edge Detection Using Expansion Matching and Restoration, IEEE Transactions on Pattern Analysis and Machine Intelligence 16 (1994) 1169-82.

[42] T. F. Cootes, A. Hill, C. J. Taylor, J. Haslam, The Use of Active Shape Model for Locating Structures in Medical Images, Image and Vision Computing 12 (1994) 355-66.

[43] A. Hill, C. J. Taylor, A. D. Brett, A Framework for Automatic Landmark Identification Using A New Method of Nonrigid Correspondence, IEEE Transactions on Pattern Analysis and Machine Intelligence 22 (2000) 241-51.

[44] D. Shen, Y. Zhan, C. Davatzikos, Segmentation of Prostate Boundaries from Ultrasound Images Using Statistical Shape Model, IEEE Transactions on Medical Imaging 22 (2003) 539-51.

[45] N. Betrouni, M. Vermandel, D. Pasquier, S. Maouche, J. Rousseau, Segmentation of Abdominal Ultrasound Images of the Prostate Using A priori Information and an Adapted Noise Filter, Computerized Medical Imaging and Graphics 29 (2005) 43-51.

[46] A. C. Hodge, A. Fenster, D. B. Downey, H. M. Ladak, Prostate Boundary Segmentation from Ultrasound Images Using 2D Active Shape Models: Optimisation and Extension to 3D, Computer Methods and Programs in Biomedicine 84 (2006) 99-113.

[47] Y. Zhu, S. Williams, R. Zwiggelaar, A Hybrid ASM Approach for Sparse Volumetric Data Segmentation, Pattern Recognition and Image Analysis 17 (2007) 252-8.

[48] X. Tang, Y. Jeong, R. J. Radke, G. T. Y. Chen, Geometric Model Based Segmentation of the Prostate and Surrounding Structures for Image Guided Radiotherapy, in: Proceedings of the SPIE Medical Imaging : Visual Communications and Image Processing, SPIE, USA, 2004, pp. 168-76.

[49] Q. Feng, M. Foskey, S. Tang, W. Chen, D. Shen, Segmenting ct prostate images using population and patient-specific statistics for radiotherapy, in: Biomedical Imaging: From Nano to Macro, ISBI, pp. 282 -5.

[50] D. G. Lowe, Object Recognition from Local Scale-Invariant Features, in: The Proceedings of the Seventh IEEE International Conference on Computer Vision, IEEE Computer Society Press, USA, 1999, pp. 1150-7.

[51] S. Osher, J. A. Sethian, Fronts Propagating With Curvature-Dependent Speed: Algorithms based on Hamilton-Jacobi Formulation, Journal of Computational Physics 79 (1988) 12-49.

[52] N. N. Kachouie, P. Fieguth, S. Rahnamayan, An Elliptical Level Set Method for Automatic TRUS Prostate Image Segmentation, in: 29th Annual International Conference of the IEEE Engineering in Medicine and Biology Society, IEEE Computer Society Press, USA, 2006, pp. $191-6$.

[53] N. N. Kachouie, P. Fieguth, A Medical Texture Local Binary Pattern For TRUS Prostate Segmentation, in: Proceedings of the 29th Annual International Conference of the IEEE Engineering in Medicine and Biology Society, IEEE Computer Society Press, USA, 2007, pp. 5605-8.

[54] N. Hu, D. B. Downey, A. Fenster, H. M. Ladak, Prostate Surface Segmentation from 3D Ultrasound Images, in: Proceedings IEEE International Symposium on Biomedical Imaging, IEEE Computer Society Press, USA, 2002, pp. 613-6.

[55] M. Ding, I. Gyacskov, X. Yuan, M. Drangova, D. Downey, A. Fenster, Slice-Based Prostate Segmentation in 3D US Images Using Continuity Constraint, in: Proceedings of 27th Annual International Conference of the Engineering in Medicine and Biology Society, IEEE Computer 
Society Press, USA, 2006, pp. 662-5.

[56] S. Badiei, S. E. Salcudean, J. Varah, W. J. Morris, Prostate Segmentation in 2D Ultrasound Images Using Image Warping and Ellipse Fitting, in: R. Larsen, M. Nielsen, J. Sporring (Eds.), Medical Image Computing and Computer-Assisted Intervention - MICCAI, Springer, Berlin and Heidelberg and New York, 2006, pp. 17-24.

[57] L. Saroul, O. Bernard, D. Vray, D. Friboulet, Prostate Segmentation in Echographic Images: A Variational Approach Using Deformable Super-Ellipse and Rayleigh Distribution, in: 5th IEEE International Symposium on Biomedical Imaging: From Nano to Macro, IEEE Computer Society Press, USA, 2008, pp. 129-32.

[58] S. Mahdavi, N. Chng, I. Spadinger, W. J. Morris, S. E. Salcudeana, Semi-Automatic Segmentation for Prostate Interventions, Medical Image Analysis 15 (2011) 226-37.

[59] M. Cabezas, A. Oliver, X. Lladó, J. Freixenet, M. B. Cuadra, A review of atlas-based segmentation for magnetic resonance brain images, Computer Methods and Programs in Biomedicine 104 (2011) 158-77.

[60] S. Klein, U. A. van der Heide, I. M. Lipps, M. V. Vulpen, M. Staring, J. P. W. Pluim, Automatic Segmentation of the Prostate in 3D MR Images by Atlas Matching Using Localized Mutual Information, Medical Physics 35 (2008) 1407-17.

[61] D. Rueckert, P. Aljabar, R. A. Heckemann, J. V. Hajnal, A. Hammers, Diffeomorphic Registration Using B-Splines, in: R. Larsen, M. Nielsen, J. Sporring (Eds.), Medical Image Computing and Computer-Assisted Intervention MICCAI, Springer, Berlin and Heidelberg and New York and Hong Kong and London and Milan and Paris and Tokyo, 2006, pp. 702-9.

[62] J. Dowling, J. Fripp, S. Chandra, J. P. W. Pluim, J. Lambert, J. Parker, J. Denham, P. B. Greer, O. Salvado, Fast automatic multi-atlas segmentation of the prostate from $3 \mathrm{~d} \mathrm{mr}$ images, in: A. Madabhushi, J. Dowling, H. J. Huisman, D. C. Barratt (Eds.), Prostate Cancer Imaging, volume 6963 of Lecture Notes in Computer Science, Springer, 2011, pp. 10-21.

[63] R. Langerak, U. A. van der Heide, A. N. T. J. Kotte, M. A. Viergever, M. van Vulpen, J. P. W. Pluim, Label fusion in atlas-based segmentation using a selective and iterative method for performance level estimation (simple), IEEE Trans. Med. Imaging 29 (2010) 2000-8.

[64] O. Acosta, J. Dowling, G. Cazoulat, A. Simon, O. Salvado, R. de Crevoisier, P. Haigron, Atlas based segmentation and mapping of organs at risk from planning ct for the development of voxel-wise predictive models of toxicity in prostate radiotherapy, in: A. Madabhushi, J. Dowling, P. Yan, A. Fenster, P. Abolmaesumi, N. Hata (Eds.), Prostate Cancer Imaging, volume 6367 of Lecture Notes in Computer Science, Springer, 2010, pp. 42-51.

[65] O. Acosta, A. Simon, F. Monge, F. Commandeur, C. Bassirou, G. Cazoulat, R. de Crevoisier, P. Haigron, Evaluation of multi-atlas-based segmentation of ct scans in prostate cancer radiotherapy, in: ISBI, IEEE, 2011, pp. 1966-9.

[66] Y. Boykov, O. Veksler, Graph Cuts in Vision and Graphics: Theories and Applications, In Handbook of Mathematical Models in Computer Vision, Springer, USA, 2006. Editors: Nikos Paragios and Yunmei Chen and Oliver D. Faugeras.

[67] M. Zouqi, J. Samarabandu, Prostate Segmentation from 2D Ultrasound Images Using Graph Cuts and Domain Knowledge, in: Canadian Conference on Computer and Robot Vision, IEEE Computer Society Press, USA, 2008, pp. 359-62.

[68] Y. Boykov, V. Kolmogorov, An Experimental Comparison of Min-cut/Max-flow Algorithms for Energy Minimization in Vision, IEEE Transactions on Pattern Analysis and Machine Intelligence 26 (2004) 1124-37.

[69] T. F. Chan, L. A. Vese, Active Contours Without Edges, IEEE Transactions on Image Processing 10 (2001) 266-77.

[70] S. Fan, L. Voon, N. Sing, 3D Prostate Surface Detection from Ultrasound Images Based on Level Set Method, in: T. Dohi, R. Kikinis (Eds.), Medical Image Computing and Computer-Assisted Intervention MICCAI, Springer, Berlin and Heidelberg and New York and Hong Kong and London and Milan and Paris and Tokyo, 2002, pp. 389-96.

[71] A. Zaim, Automatic Segmentation of the Prostate from Ultrasound Data Using Feature-Based Self Organizing Map, in: H. Kalviainen, J. Parkkinen, A. Kaarna (Eds.), Proceedinggs of Scandinavian Conference in Image Analysis, Springer, Berlin and Heidelberg and New York, 2005, pp. 1259-65.

[72] A. Zaim, Y. Taeil, R. Keck, Feature Based Classification of Prostate US Images Using Multiwavelet and Kernel SVM, in: Proceedings of International Joint Conference on Neural Networks, IEEE Computer Society Press, USA, 2007, pp. 278-81.

[73] J. S. Geronimo, D. P. Hardin, P. R. Massopust, Fractal Functions and Wavelet Expansions Based on Several Scaling Functions, Journal of 
Approximation Theory 78 (1994) 373-401.

[74] S. S. Mohamed, A.M.Youssef, E. F. El-Saadany, M. M. A. Salama, Prostate Tissue Characterization Using TRUS Image Spectral Features, Springer, Berlin and Heidelberg and New York, 2006, pp. 589-601.

[75] T. Randen, J. H. Husoy, Filtering for Texture Classification: A Comparative Study, Transactions on Pattern Analysis and Machine Intelligence 21 (1999) $291-310$.

[76] W. D. Richard, C. G. Keen, Automated Texture Based Segmentation of Ultrasound Images of the Prostate, Computerized Medical Imaging and Graphics 20 (1996) 131-40.

[77] D. Comaniciu, P. Meer, Mean Shift: A Robust Approach Toward Feature Space Analysis, IEEE Transactions on Pattern Analysis and Machine Intelligence 24 (2002) 603-19.

[78] H. Liu, G. Cheng, D. Rubens, J. G. Strang, L. Liao, R. Brasacchio, E. Messing, Y. Yu', Automatic Segmentation of Prostate Boundaries in Transrectal Ultrasound (TRUS) Imaging, in: M. Sonka, J. M. Fitzpatrick (Eds.), Proceedings of the SPIE Medical Imaging : Image Processings, SPIE, USA, 2002, pp. 412-23.

[79] P. Yan, S. Xu, B. Turkbey, J. Kruecker, Discrete Deformable Model Guided by Partial Active Shape Model for TRUS Image Segmentation, IEEE Transactions on Biomedical Engineering 57 (2010) 1158-66.

[80] Y. Zhan, D. Shen, Automated Segmentation of 3D US Prostate Images Using Statistical Texture-Based Matching Method, in: R. E. Ellis, T. M. Peters (Eds.), Medical Image Computing and Computer-Assisted Intervention - MICCAI, Springer, Berlin and Heidelberg and New York, 2003, pp. 688-96.

[81] Y. Zhan, D. Shen, An Efficient Method for Deformable Segmentation of 3D US Prostate Images, in: G.-Z. Yang, T.-Z. Jiang (Eds.), Second International Workshop on Medical Imaging and Augmented Reality, Springer, Berlin and Heidelberg and New York, 2004, pp. 103-12.

[82] S. Ghosal, R. Mehrotra, Orthogonal Moment Operators for Subpixel Edge Detection, Pattern Recognition 26 (1993) $295-306$.

[83] Y. Zhan, D. Shen, Increasing Efficiency of SVM by Adaptively Penalizing Outliers, in: A. Rangarajan, B. Vemuri, A. L. Yuille (Eds.), 5th International Workshop on Energy Minimization Methods in Computer Vision and Pattern Recognition, Springer, Berlin and Heidelberg and New York, 2005, pp. 539-51.

[84] K. Diaz, B. Castaneda, Semi-automated Segmentation of the Prostate Gland Boundary in Ultrasound Images Using a Machine Learning Approach, in: J. M. Reinhardt, J. P. W. Pluim (Eds.), Procedings of SPIE Medical Imaging : Image Processing, SPIE, USA, 2008 , pp. 1-8.

[85] R. Medina., A. Bravo, P. Windyga, J. Toro, P. Yan, G. Onik, A 2D Active Appearance Model For Prostate Segmentation in Ultrasound Images, in: 27th Annual International Conference of the IEEE Engineering in Medicine and Biology Society, IEEE Computer Society Press, USA, 2005, pp. 3363-6.

[86] T. Cootes, G. Edwards, C. Taylor, Active Appearance Models, in: H.Burkhardt, B. Neumann (Eds.), In Proceedings of European Conference on Computer Vision, Springer, Berlin and Heidelberg and New York, 1998, pp. 484-98.

[87] S. Ghose, A. Oliver, R. Martí, X. Lladó, J. Freixenet, J. C. Vilanova, F. Meriaudeau, Texture Guided Active Appearance Model Propagation for Prostate Segmentation, in: A. Madabhushi, J. Dowling, P. Yan, A. Fenster, P. Abolmaesumi, N. Hata (Eds.), Prostate Cancer Imaging, volume 6367 of Lecture Notes in Computer Science, Springer, 2010, pp. 111-20.

[88] S. Ghose, A. Oliver, R. Martí, X. Lladó, J. Freixenet, J. Mitra, J. C. Vilanova, J. Comet, F. Meriaudeau, Statistical shape and texture model of quadrature phase information for prostate segmentation, International Journal of Computer Assisted Radiology and Surgery 7 (2012) 43-55.

[89] S. Ghose, A. Oliver, R. Martí, X. Lladó, J. Freixenet, J. Mitra, J. C. Vilanova, J. Comet, F. Meriaudeau, Multiple Mean Models of Statistical Shape and Probability Priors for Automatic Prostate Segmentation, in: A. Madabhushi, J. Dowling, H. J. Huisman, D. C. Barratt (Eds.), Prostate Cancer Imaging, volume 6963 of Lecture Notes in Computer Science, Springer, 2011, pp. 35-46.

[90] L. Gong, S. D. Pathak, D. R. Haynor, P. S. Cho, Y. Kim, Parametric Shape Modeling Using Deformable Superellipses for Prostate Segmentation, IEEE Transactions on Medical Imaging 23 (2004) 340-9.

[91] I. B. Tutar, S. D. Pathak, L. Gong, P. S. Cho, K. Wallner, Y. Kim, Semiautomatic 3D Prostate Segmentation from TRUS Images Using Spherical Harmonics, IEEE Transactions on Medical Imaging 25 (2006) 1645-54. 
[92] F. Wang, J. Suri, A. Fenster, Segmentation of Prostate from 3D Ultrasound Volumes Using Shape and Intensity Priors in Level Set Framework, Proceedings of the 28th IEEE Engineering in Medicine and Biology Society (2006) 2341-4.

[93] R. Malladi, J. A. Sethian, Image Processing via Level set Curvature Flow, Proceedings of the National Academy of Sciences 92 (1995) $7046-50$.

[94] C. Garnier, J.-J. Bellanger, K. Wu, H. Shu, N. Costet, R. Mathieu, R. de Crevoisier, J.-L. Coatrieux, Prostate segmentation in HIFU therapy, IEEE Trans. Medical Imaging 30 (2011) 792-803.

[95] S. Vikal, S. Haker, C. Tempany, G. Fichtinger, Prostate Contouring in MRI Guided Biopsy, in: J. P. W. Pluim, B. M. Dawant (Eds.), Proceedings of SPIE Medical Imaging: Image Processing, SPIE, USA, 2009, pp. 7259-72594A.

[96] P. D. Allen, J. Graham, D. C. Williamson, C. E. Hutchinson, Differential Segmentation of the Prostate in MR Images Using Combined 3D Shape Modelling and Voxel Classification, in: 3rd IEEE International Symposium on Biomedical Imaging: Nano to Macro, IEEE Computer Society Press, USA, 2006, pp. 410-3.

[97] S. Martin, J. Troccaz, V. Daanen, Automated Segmentation of the Prostate in 3D MR Images Using a Probabilistic Atlas and a Spatially Constrained Deformable Model, Medical Physics 37 (2010) 1579 -90.

[98] A. Tsai, J. Anthony Yezzi, W. Wells, C. Tempany, D. Tucker, A. Fan, W. E. Grimson, A. Willsky, A Shape-Based Approach to the Segmentation of Medical Imagery Using Level Sets, IEEE Transactions on Medical Imaging 22 (2003) 137-54.

[99] A. Tsai, W. M. Wells, C. Tempany, E. Grimson, A. S. Willsky, Coupled Multi-shape Model and Mutual Information for Medical Image Segmentation, in: C. Taylor, J. A. Noble (Eds.), International Conference, Information Processing in Medical Imaging, Springer, Berlin and Heidelberg and New York, 2003, pp. 185-97.

[100] N. Otsu, A Threshold Selection Method from Gray-Level Histograms Systems, IEEE Transactions on System, Man and Cybernetics 9 (1979) 62-6.

[101] A. Firjani, A. Elnakib, A. El-Baz, G. L. Gimel'farb, M. A. El-Ghar, A. Elmaghraby, Novel stochastic framework for accurate segmentation of prostate in dynamic contrast enhanced mri, in: A. Madabhushi, J. Dowling, P. Yan, A. Fenster, P. Abolmaesumi, N. Hata (Eds.), Prostate Cancer Imaging, volume 6367 of Lecture Notes in Computer Science, Springer, 2010, pp. 121-30.

[102] A. Firjani, A. Elnakib, F. Khalifa, G. L. Gimel'farb, M. A. El-Ghar, J. Suri, A. Elmaghraby, A. El-Baz, A new 3d automatic segmentation framework for accurate segmentation of prostate from dce-mri, in: International Symposium on Biomedical Imaging: From Nano to Macro, IEEE, 2011, pp. 1476-9.

[103] Y. Zhang, B. J. Matuszewski, A. Histace, F. Precioso, J. Kilgallon, C. J. Moore, Boundary delineation in prostate imaging using active contour segmentation method with interactively defined object regions, in: A. Madabhushi, J. Dowling, P. Yan, A. Fenster, P. Abolmaesumi, N. Hata (Eds.), Prostate Cancer Imaging, volume 6367 of Lecture Notes in Computer Science, Springer, 2010, pp. 131-42.

[104] Y. Gao, R. Sandhu, G. Fichtinger, A. R. Tannenbaum, A Coupled Global Registration and Segmentation Framework with Application to Magnetic Resonance Prostate Imagery, IEEE Transactions on Medical Imaging 10 (2010) 17-81.

[105] R. Toth, B. N. Bloch, E. M. Genega, N. M. Rofsky, R. E. Lenkinski, M. A. Rosen, A. Kalyanpur, S. Pungavkar, A. Madabhushi, Accurate prostate volume estimation using multifeature active shape models on t2-weighted mr, Academic Radiology 18 (2011) 745 -54.

[106] R. Toth, P. Tiwari, M. Rosen, G. Reed, J. Kurhanewicz, A. Kalyanpur, S. Pungavkar, A. Madabhushi, A magnetic resonance spectroscopy driven initialization scheme for active shape model based prostate segmentation, Medical Image Analysis 15 (2011) $214-25$.

[107] P. Khurd, L. Grady, K. Gajera, M. Diallo, P. Gall, M. Requardt, B. Kiefer, C. Weiss, A. Kamen, Facilitating 3d spectroscopic imaging through automatic prostate localization in mr images using random walker segmentation initialized via boosted classifiers, in: A. Madabhushi, J. Dowling, H. J. Huisman, D. C. Barratt (Eds.), Prostate Cancer Imaging, volume 6963 of Lecture Notes in Computer Science, Springer, 2011, pp. 47-56.

[108] L. Grady, Random walks for image segmentation, IEEE Trans. Pattern Anal. Mach. Intell. 28 (2006) $1768-83$.

[109] D. Freedman, R. J. Radke, T. Zhang, Y. Jeong, D. M. Lovelock, G. T. Y. Chen, Model-Based Segmentation of Medical Imagery by Matching Distributions, IEEE Transactions on Medical Imaging 24 (2005) 281-92.

[110] M. Rousson, A. Khamene, M. Diallo, J. C. Celi, F. Sauer, Constrained Surface Evolutions for Prostate and Bladder Segmentation in CT 
Images, in: Y. Liu, T. Jiang, C. Zhang (Eds.), First International Workshop, Computer Vision for Biomedical Image Applications, Springer, Berlin and Heidelberg and New York, 2005, pp. 251-60.

[111] B. C. Davis, M. Foskey, J. Rosenman, L. Goyal, S. Chang, S. Joshi, Automatic segmentation of intra-treatment ct images for adaptive radiation therapy of the prostate, in: J. S. Duncan, G. Gerig (Eds.), Medical Image Computing and Computer-Assisted InterventionMICCAI, volume 3749 of Lecture Notes in Computer Science, Springer, 2005, pp. 442-50.

[112] P. Ghosh, M. Mitchell, Prostate Segmentation on Pelvic CT Images Using a Genetic Algorithm, in: J. M. Reinhardt, J. P. W. Pluim (Eds.), Proceedings of SPIE Medical Imaging : Image Processing, SPIE, USA, 2008, pp. 691442.1-8.

[113] M. J. Costa, D. Hervé, N. Sébastien, A. Nicholas, Automatic Segmentation of Bladder and Prostate Using Coupled 3D Deformable Models, in: N. Ayache, S. Ourselin, A. Maeder (Eds.), Medical Image Computing and Computer-Assisted Intervention MICCAI, Springer, Berlin and Heidelberg and New York, 2007, pp. 252-60.

[114] Q. Song, X. Wu, Y. Liu, M. Smith, J. Buatti, M. Sonka, Optimal Graph Search Segmentation Using Arc-Weighted Graph for Simultaneous Surface Detection of Bladder and Prostate, in: G.-Z. Yang, D. Hawkes, D. Rueckert, A. Noble, C. Taylor (Eds.), Medical Image Computing and Computer-Assisted Intervention MICCAI, Springer, Berlin and Heidelberg and New York, 2009, pp. 827-35.

[115] K. Li, M. P. Jolly, Simultaneous Detection of Multiple Elastic Surfaces with Application to Tumor Segmentation in CT Images, in: J. M. Reinhardt, J. P. W. Pluim (Eds.), Proceedings of SPIE Medical Imaging : Image Processing, SPIE, USA, 2008, pp. 69143S.1-11.

[116] Q. Song, Y. Liu, Y. Liu, P. K. Saha, M. Sonka, X. Wu, Graph search with appearance and shape information for 3-d prostate and bladder segmentation, in: T. Jiang, N. Navab, J. P. Pluim, M. A. Viergever (Eds.), Medical Image Computing and Computer-Assisted Intervention MICCAI, Springer, Berlin and Heidelberg and New York, 2010, pp. 172-80.

[117] T. Chen, S. Kim, J. Zhou, D. Metaxas, G. Rajagopal1, N. Yue, 3D Meshless Prostate Segmentation and Registration in Image Guided Radiotherapy, in: G.-Z. Yang, D. Hawkes, D. Rueckert, A. Noble, C. Taylor (Eds.), Medical Image Computing and Computer-Assisted Intervention MICCAI, Springer, Berlin and Heidelberg and New York, 2009, pp. 43-50.

[118] S. Chen, R. J. Radhke, Level Set Segmentation with Both Shape and Intensity Priors, in: International Conference on Computer Vision, IEEE Computer Society Press, USA, 2009, pp. 763-70.

[119] Q. Feng, M. Foskey, S. Tang, W. Chen, D. Shen, Segmenting CT Prostate Images Using Population and Patient-Specific Statistics for Radiotherapy, Medical Physics 37 (2010).

[120] W. Li, S. Liao, Q. Feng, W. Chen, D. Shen, Learning image context for segmentation of prostate in ct-guided radiotherapy, in: G. Fichtinger, A. L. Martel, T. M. Peters (Eds.), Medical Image Computing and Computer-Assisted Intervention - MICCAI, volume 6893 of Lecture Notes in Computer Science, Springer, 2011, pp. 570-8.

[121] S. Liao, D. Shen, A learning based hierarchical framework for automatic prostate localization in ct images, in: A. Madabhushi, J. Dowling, H. J. Huisman, D. C. Barratt (Eds.), Prostate Cancer Imaging, volume 6963 of Lecture Notes in Computer Science, Springer, 2011, pp. 1-9.

[122] C. Lu, S. Chelikani, X. Papademetris, J. Knisely, M. F. Milosevic, Z. Chen, D. A. Jaffray, L. H. Staib, J. S. Duncan, An integrated approach to segmentation and nonrigid registration for application in image-guided pelvic radiotherapy, Medical Image Analysis 15 (2011) $772-85$.

[123] S. Chen, D. M. Lovelock, R. J. Radke, Segmenting the prostate and rectum in ct imagery using anatomical constraints, Medical Image Analysis 15 (2011) 1-11.

[124] E. Belogay, C. Cabrelli, U. Molter, R. Shonkwiler, Calculating the hausdorff distance between curves, Information Processing Letters 64 (1997) 17-22.

[125] MICCAI, 2009 prostate segmentation challenge MICCAI, http://wiki.na-mic.org/Wiki/index.php, accessed on [1st April, 2011$], 2009$.

[126] C. Rasch, I. Barillot, P. Remeijer, A. Touw, M. van Herk, J. V. Lebesque, Definition of the prostate in ct and mri: a multi-observer study, International Journal of Radiation Oncology*Biology*Physics 43 (1999) 57 - 66.

[127] Prostate MRI database, http://prostatemriimagedatabse.com, accessed on [12th Jan, 2012], 2012.

[128] S. Xu, J. Kruecker, B. Turkbey, N. Glossop, A. Singh, P. Chyke, P. Pinto, B. Wood, Real-time MRI/TRUS Fusion for Guidance of Targeted Prostate Biopsies., Computer Aided Surgery 13 (2008) 255-64.

[129] S. Viswanath, D. Palumbo, J. Chappelow, P. Patel, B. N. Bloch, N. NRofsky, R. Lenkinski, E. Genega, A. Madabhushi, Empirical evaluation 
of bias field correction algorithms for computer aided detection of prostate cancer on t2w mri, in: SPIE Medical Imaging, volume 7963, SPIE, SPIE, 2011, pp. 79630V-12.

[130] J. G. Sled, A. P. Zijdenbos, A. C. Evans, A nonparametric method for automatic correction of intensity nonuniformity in mri data, IEEE Transactions on Medical Imaging 17 (1998) 87-97.

[131] M. Styner, C. Brechbuhler, G. Szckely, G. Gerig, Parametric estimate of intensity inhomogeneities applied to mri, IEEE Transactions on Medical Imaging 19 (2000) $153-65$.

[132] M. S. Cohen, R. M. DuBois, M. M. Zeineh, Rapid and effective correction of rf inhomogeneity for high field magnetic resonance imaging, Human Brain Mapping 10 (2000) $204-11$.

[133] J. Dowling, J. Fripp, P. Greer, S. Ourselin, O. Salvado, Automatic atlas-based segmentation of the prostate, www.wiki.namic.org/Wiki/images/f/f1/Dowling_2009 _MICCAIProstate_v2.pdf, Accessed on [20th Jan, 2012], 2009.

[134] W. Fledelius, E. Worm, C. Grau, M. Hyer, U. V. Elstrm, J. B. Petersen, P. R. Poulsen, Segmenting the prostate and rectum in ct imagery using anatomical constraints, Medical Physics 38 (2011) 6351-61.

[135] P. Slagmolen, J. Hermans, F. Maes, T. Budiharto, K. Haustermans, F. V. D. Heuvel, Fast, accurate, and robust automatic marker detection for motion correction based on oblique kv or mv projection image pairs, Medical Physics 37 (2010) 1554-5.

[136] A. C. Hodge, H. M. Ladak, 3D Prostate Boundary Segmentation From Ultrasound Images Using 2D Active Shape Models, in: 28th Annual International Conference of the IEEE Engineering in Medicine and Biology Society, IEEE Computer Society Press, USA, 2006, pp. 2337-40.

[137] L. Gong, L. Ng, S. D. Pathak, I. Tutar, P. S. Choc, D. R. Haynord, Y. Kim, Prostate Ultrasound Image Segmentation Using Level set-Based Region Flow With Shape Guidance, in: J. M. Fitzpatrick, J. M. Reinhardt (Eds.), Proceedings of SPIE Medical Imaging : Image Processing, SPIE, USA, 2005, pp. 1648-57. 


\section{List of Figures}

1 TRUS, MRI and CT provide different information of the prostate (the contour is outlined in green). TRUS and MRI images of the same patient. (a) In TRUS imaging, the prostate is shown as a hypoechoic mass surrounded by a hyperechoic halo. (b) T2 weighted MRI allows to see the internal anatomy of the prostate. Note that the contrast around prostate is enhanced with endorectal coil (A). (c) CT image of a different prostate showing radioactive seeds in white. . . . . . . . . . . . . . 34

2 Our proposed taxonomy of prostate segmentation. TRUS references in red, MRI references in green and $\mathrm{CT}$ references in blue . . . . . . . . . . . . . . . . . . . . . . 35 


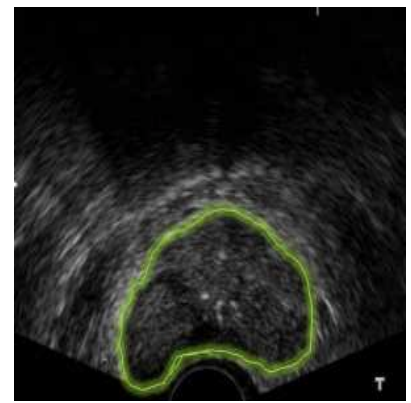

(a)

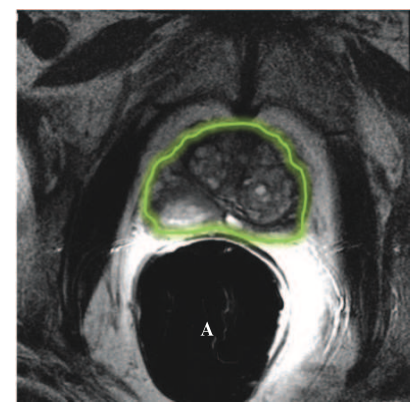

(b)

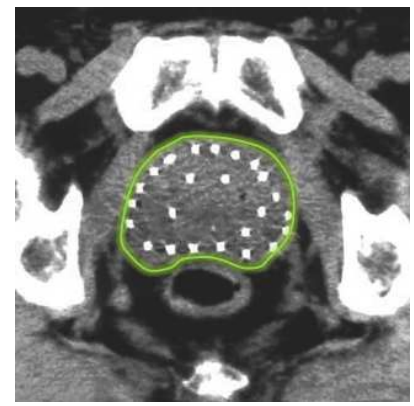

(c)

Figure 1: TRUS, MRI and CT provide different information of the prostate (the contour is outlined in green). TRUS and MRI images of the same patient. (a) In TRUS imaging, the prostate is shown as a hypoechoic mass surrounded by a hyperechoic halo. (b) T2 weighted MRI allows to see the internal anatomy of the prostate. Note that the contrast around prostate is enhanced with endorectal coil (A). (c) CT image of a different prostate showing radioactive seeds in white. 


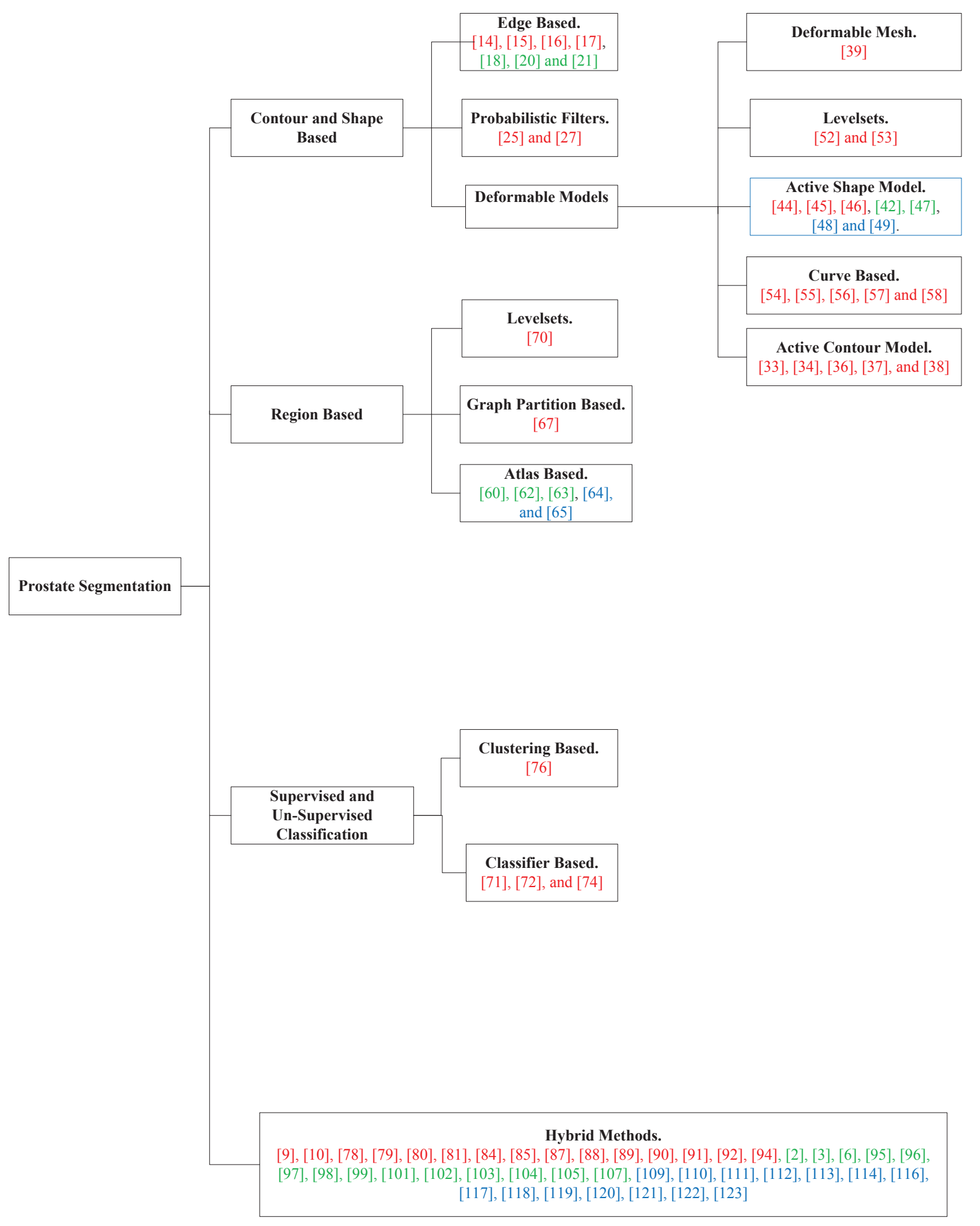

Figure 2: Our proposed taxonomy of prostate segmentation. TRUS references in red, MRI references in green and CT references in blue 


\section{List of Tables}

1 Advantages and disadvantages of the prostate imaging techniques. . . . . . . . . . . . . . . 37

2 Advantages and disadvantages of the reviewed prostate segmentation approaches . . . . . . . . 38

3 Evaluation metrics for 2D . . . . . . . . . . . . . . . . . . . . . 39

4 Evaluation metrics for 3D segmentation . . . . . . . . . . . . . . . . . . 40

5 Quantitative evaluations : prostate segmentation in TRUS imaging . . . . . . . . . . . . . . 41

6 Quantitative evaluations : prostate segmentation in TRUS imaging continued... . . . . . . . . . . . 42

7 Quantitative evaluations : prostate segmentation in MR images . . . . . . . . . . . . . . . . . . 43

8 Quantitative evaluations : prostate segmentation in CT images . . . . . . . . . . . . . . . . . . 44 
Table 1: Advantages and disadvantages of the prostate imaging techniques.

\begin{tabular}{|c|c|c|}
\hline & Advantages & Disadvantages \\
\hline TRUS & $\begin{array}{l}\text { Useful in determining prostate volume } \\
\text { No radiation involved } \\
\text { Inexpensive } \\
\text { Portable } \\
\text { Useful for real time imaging }\end{array}$ & $\begin{array}{l}\text { Low contrast images } \\
\text { Difficult to detect lesions } \\
\text { Speckle } \\
\text { Shadow artifacts } \\
\text { Cancer staging is difficult } \\
\end{array}$ \\
\hline MRI & $\begin{array}{l}\text { Useful in determining prostate volume } \\
\text { No radiation involved } \\
\text { High contrast for soft-tissues } \\
\text { Allows lesion detection } \\
\text { Enables functional imaging of prostate } \\
\text { Staging of cancer possible }\end{array}$ & $\begin{array}{l}\text { Expensive } \\
\text { Not portable } \\
\text { Difficult to implement real time imaging }\end{array}$ \\
\hline CT & $\begin{array}{l}\text { Useful in determining spread of prostate cancer to bone tissues } \\
\text { Useful in determining effectiveness of prostate brachytherapy }\end{array}$ & $\begin{array}{l}\text { Expensive } \\
\text { Radiation involved } \\
\text { Not portable } \\
\text { Poor soft-tissue contrast } \\
\text { Difficult to detect lesions } \\
\text { Cancer staging is difficult } \\
\text { Difficult to implement real time imaging }\end{array}$ \\
\hline
\end{tabular}


Table 2: Advantages and disadvantages of the reviewed prostate segmentation approaches

\begin{tabular}{|c|c|c|c|c|}
\hline & & Approaches & Advantages & Disadvantages \\
\hline \multirow{8}{*}{ 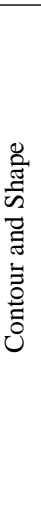 } & \multicolumn{2}{|r|}{ Edge } & Easy to extract & Edge information is unreliable and often broken \\
\hline & \multicolumn{2}{|r|}{ Shape } & $\begin{array}{l}\text { Provides robustness against noise and arti- } \\
\text { facts }\end{array}$ & Depends on strong edge information for fitting \\
\hline & \multicolumn{2}{|r|}{ Probabilistic filters } & Robust against noise along boundary & Difficult to initialize and to extend to 3D \\
\hline & \multirow{5}{*}{ 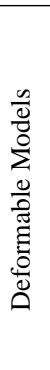 } & $\mathrm{ACM}$ & $\begin{array}{l}\text { Easy to implement, produces smooth con- } \\
\text { tours }\end{array}$ & $\begin{array}{l}\text { Depends on reliable edge information, good initial- } \\
\text { ization required, large-scale deformations produce } \\
\text { spurious corners }\end{array}$ \\
\hline & & Mesh & Shape information is preserved & $\begin{array}{l}\text { Reliable edge information is often necessary, rigid } \\
\text { shape representation, slow in speed }\end{array}$ \\
\hline & & ASM & $\begin{array}{l}\text { Shape representation and variation in Gaus- } \\
\text { sian space is defined }\end{array}$ & $\begin{array}{l}\text { Inaccurate in large-scale shape variations, extension } \\
\text { to } 3 \mathrm{D} \text { is difficult, need of training }\end{array}$ \\
\hline & & Contour level set & $\begin{array}{l}\text { Contour implicitly defined, easy extension to } \\
\text { 3D }\end{array}$ & Depends on reliable edge information, slow in speed \\
\hline & & Curve fitting & Easy to implement, fast & $\begin{array}{l}\text { Rigid shape structure, reliable edge information is } \\
\text { necessary }\end{array}$ \\
\hline ซี & & Atlas & $\begin{array}{l}\text { Automatic, robust to contrast differences, in- } \\
\text { corporate prior shape and intensity informa- } \\
\text { tion }\end{array}$ & $\begin{array}{l}\text { Building atlas is not trivial and prone to registration } \\
\text { errors, slow in speed of segmentation }\end{array}$ \\
\hline 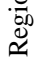 & & Graph partitioning & $\begin{array}{l}\text { Efficient optimization, region based informa- } \\
\text { tion could be incorporated }\end{array}$ & $\begin{array}{l}\text { Incorporating shape priors is difficult, manual interac- } \\
\text { tion often necessary }\end{array}$ \\
\hline & & Region level set & $\begin{array}{l}\text { Region based information more reliable than } \\
\text { edge, implicit contour }\end{array}$ & $\begin{array}{l}\text { Intensity heterogeneity produces fragmented regions, } \\
\text { no prior shape information, slow in speed }\end{array}$ \\
\hline & & Clustering & Prior training not required, automatic & No prior shape information \\
\hline$\stackrel{a}{a}$ & & Classification & Robust against noise, automatic & $\begin{array}{l}\text { No prior shape information, a training step is neces- } \\
\text { sary }\end{array}$ \\
\hline$\frac{\pi}{2}$ & Cor & n of any of the above approaches & $\begin{array}{l}\text { More robust to imaging artifacts and noises, } \\
\text { produces accurate segmentations }\end{array}$ & $\begin{array}{l}\text { Choice of combining information from different } \\
\text { sources is complicated, often the methods are opti- } \\
\text { mized for prostate segmentation and less generic }\end{array}$ \\
\hline
\end{tabular}


Table 3: Evaluation metrics for 2D

\begin{tabular}{|c|c|c|c|c|}
\hline & Metric & Parameters & Equation & Used by \\
\hline \multirow{5}{*}{ 泀 } & Hausdorff distance (HD) & \multirow[t]{2}{*}{$\begin{array}{l}\text { Given a set of } \\
\text { finite } \\
A=\left\{a_{1}, a_{2}, \ldots a_{p}\right\} \text { and } \\
B=\left\{b_{1}, b_{2}, \ldots, b_{q}\right\}\end{array}$} & $\begin{array}{l}H D(A, B)=\max (h(A, b), h(B, A)) \\
\text { where } h(A, B)=\max _{a \in A}\left(\min _{b \in B}\|a-b\|\right)\end{array}$ & [17], [90] \\
\hline & Root mean square distance (RMSD) & & $\operatorname{RMSD}(A, B)=\sqrt{\frac{1}{N} \sum_{j=1}^{N}\left(A_{j}-B_{j}\right)^{2}}$ & [47] \\
\hline & Mean Distance (MD) & \multirow[t]{3}{*}{$\begin{array}{l}\text { Given signed distance } \\
d_{j} \text { between each } \\
\text { corresponding points } \\
j(j=1,2, \ldots, N) \text { be- } \\
\text { tween the algorithmic } \\
\text { segmented surface } \\
\text { and ground truth. }\end{array}$} & $M D=\frac{1}{N} \sum_{j=1}^{N} d_{j}$ & $\begin{array}{l}{[27],[33],[34],} \\
{[44],[45],[136],} \\
{[90],[54],[78],} \\
{[85],[9], \text { and }[48]}\end{array}$ \\
\hline & Mean absolute distance (MAD) & & $M A D=\frac{1}{N} \sum_{j=1}^{N}\left|d_{j}\right|$ & $\begin{array}{l}{[17],[34],[55],} \\
{[136],[54],[78],} \\
{[137],[91],[10],} \\
{[79],[88],[95],} \\
{[97][122] \text { and }} \\
{[106]}\end{array}$ \\
\hline & Maximum distance (MaxD) & & $\operatorname{Max} D=\max \left|d_{j}\right|$ & $\begin{array}{l}{[34],[45],[136],} \\
{[54],[78],[91],} \\
{[10], \text { and }[79]}\end{array}$ \\
\hline \multirow{7}{*}{ 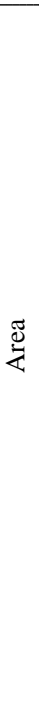 } & Dice similarity coefficient (DSC) & \multirow{6}{*}{$\begin{array}{l}\mathrm{TP}=\text { True positive, } \\
\mathrm{TN}=\text { True negative, } \\
\mathrm{FP}=\text { False positive, } \\
\text { and FN = False Neg- } \\
\text { ative }\end{array}$} & $D S C=\frac{2 T P}{(F P+T P)+(T P+F N)}$ & $\begin{array}{l}{[88],[20],[21],} \\
{[95],[6]}\end{array}$ \\
\hline & Sensitivity (SN) & & $S N=\frac{T P}{T P+F N}$ & $\begin{array}{l}{[34],[56],[74],} \\
{[88], \text { and }[113]}\end{array}$ \\
\hline & Specificity (SP) & & $S P=\frac{T N}{T N+F P}$ & {$[88]$} \\
\hline & Accuracy (AC) & & $A C=\frac{T P+T N}{T P+T N+F P+F N}$ & {$[34],[56],[74]$} \\
\hline & Overlap (OV) & & $O V=\frac{T P}{F P+F N}$ & $\begin{array}{l}{[25],[38],[45],} \\
{[71],[85]}\end{array}$ \\
\hline & Overlap Error (OE) & & $O E=1-O v$ & [44], and [27] \\
\hline & Surface distance (SD) & $\begin{array}{l}\text { Given unsigned dis- } \\
\text { tance } d_{s} \text { between } \\
\text { between the algo- } \\
\text { rithmic segmented } \\
\text { surface and ground } \\
\text { truth. }\end{array}$ & $S D=\frac{1}{N} \sum_{N}^{s=1} d_{s}$ & $\begin{array}{l}{[62],[49],[120],} \\
{[110],[109], \text { and }} \\
{[114]}\end{array}$ \\
\hline
\end{tabular}


Table 4: Evaluation metrics for 3D segmentation

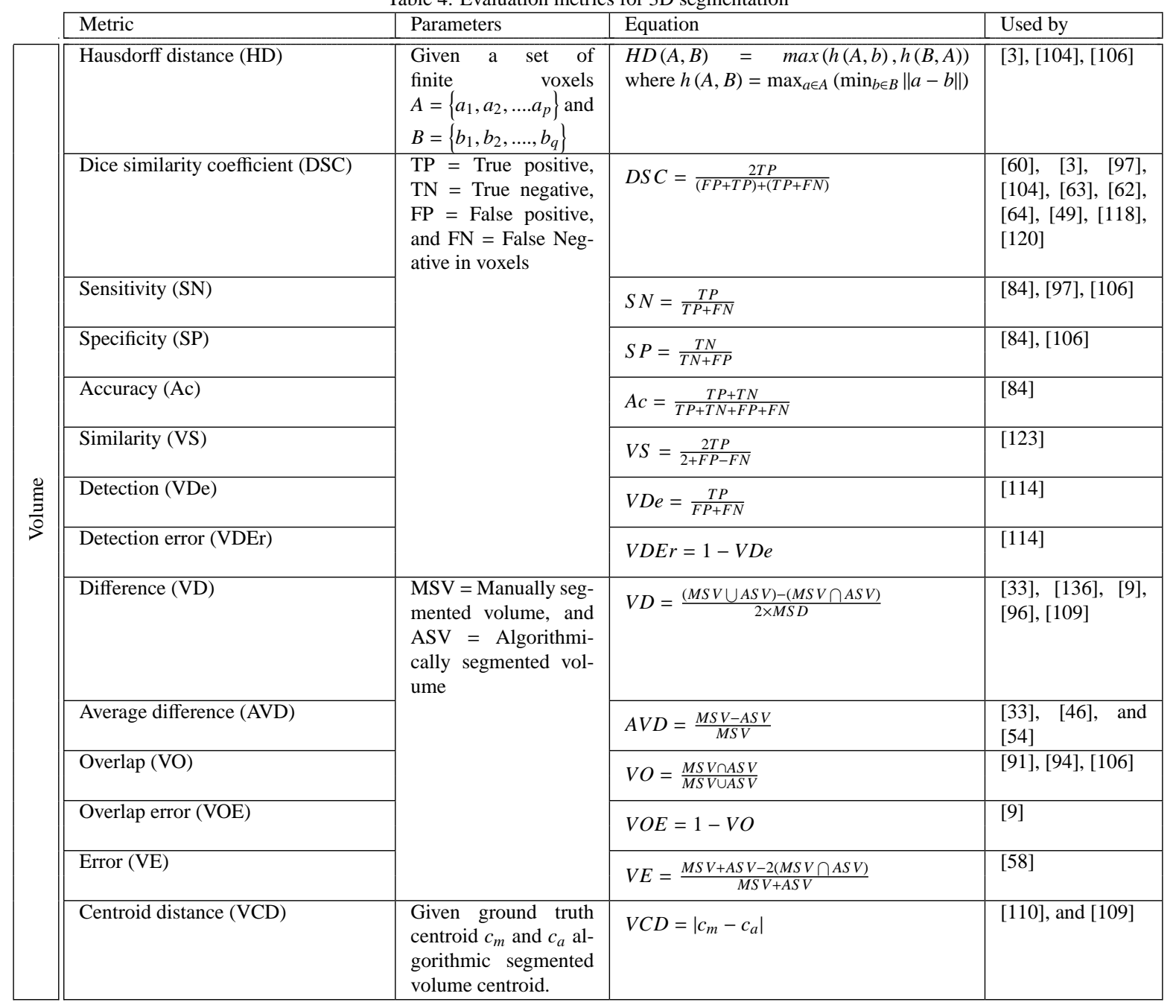


Table 5: Quantitative evaluations : prostate segmentation in TRUS imaging

\begin{tabular}{|c|c|c|c|c|c|c|c|c|c|c|}
\hline & \multirow[t]{2}{*}{ Reference } & \multirow[t]{2}{*}{ Year } & \multirow[t]{2}{*}{ Dim } & \multirow[t]{2}{*}{$\mathrm{B} / \mathrm{A}$} & \multirow[t]{2}{*}{ Pre-processing } & \multirow[t]{2}{*}{ Segmentation Criteria } & \multirow[t]{2}{*}{ Auto } & \multicolumn{2}{|c|}{ Performance } & \multirow[t]{2}{*}{ Validation } \\
\hline & & & & & & & & Measure & Value & \\
\hline \multirow{15}{*}{ 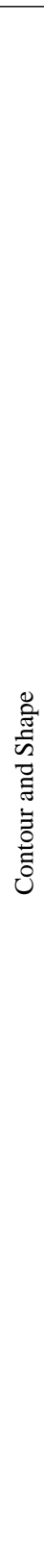 } & Pathak [17] & 2000 & $2 \mathrm{D}$ & Yes & Stick filter & Edge Based & No & $\begin{array}{l}\text { Contour MAD } \\
\text { Contour HD }\end{array}$ & $\begin{array}{l}1.5 \mathrm{~mm} \\
4 \mathrm{~mm}\end{array}$ & 125 images \\
\hline & Abolmaesumi [25] & 2004 & $2 \mathrm{D}$ & No & Stick filter & Probabilistic Filter & No & Area OV & $98 \%$ & 6 images \\
\hline & Sahba [27] & 2005 & $2 \mathrm{D}$ & No & $\begin{array}{l}\text { Smoothing, } \\
\text { morphological } \\
\text { filtering and } \\
\text { thresholding }\end{array}$ & Probabilistic Filter & No & $\begin{array}{l}\text { Contour MD } \\
\text { Area error }\end{array}$ & $\begin{array}{l}3.3 \pm 1.3 \text { pixels } \\
2.4 \pm 1.1 \%\end{array}$ & 19 images \\
\hline & Knoll [33] & 1999 & $3 \mathrm{D}$ & Yes & $\begin{array}{l}\text { Wavelet for } \\
\text { edge enhance- } \\
\text { ment }\end{array}$ & DM - ACM & Yes & $\begin{array}{l}\text { Volume VD } \\
\text { Contour MD } \\
\text { Volume AVD }\end{array}$ & $\begin{array}{l}10.97 \% \\
2.61 \mathrm{~mm} \\
8.48 \%\end{array}$ & 77 images \\
\hline & Ladak [34] & 2000 & $2 \mathrm{D}$ & No & No & DM - ACM & No & $\begin{array}{l}\text { Contour MAD } \\
\text { Contour MaxD } \\
\text { Area AC } \\
\text { Area SN }\end{array}$ & $\begin{array}{l}4.4(\approx 0.63 \mathrm{~mm}) \pm 1.8 \\
\text { pixels } \\
19.5(\approx 2.5 \mathrm{~mm}) \pm 7.8 \\
\text { pixels } \\
90.1 \pm 3.2 \% \\
94.5 \pm 2.7 \%\end{array}$ & 117 images \\
\hline & Ding [55] & 2005 & $3 \mathrm{D}$ & Yes & No & DM - ACM & No & Contour MAD & $2.79 \pm 1.94 \mathrm{~mm}$ & 6 data sets \\
\hline & Zaim [38] & 2007 & $2 \mathrm{D}$ & No & $\begin{array}{l}\text { Median and } \\
\text { morphological } \\
\text { filtering }\end{array}$ & DM - ACM & Yes & Area OV & $92 \%$ & 10 images \\
\hline & Ghanei [39] & 2001 & $3 \mathrm{D}$ & Yes & No & DM - Mesh & No & Volume VS & $89 \%$ & 10 data sets \\
\hline & Shen [44] & 2003 & $2 \mathrm{D}$ & No & Gabor features & DM - ASM & Yes & $\begin{array}{l}\text { Contour MD } \\
\text { Area OE } \\
\text { Area error } \\
\end{array}$ & $\begin{array}{l}3.2(\approx 1.28 \quad \mathrm{~mm}) \quad \pm \\
0.87 \text { pixels } \\
3.98 \pm 0.97 \% \\
1.66 \pm 1.68 \%\end{array}$ & 8 images \\
\hline & Betrouni [45] & 2004 & $2 \mathrm{D}$ & No & $\begin{array}{l}\text { Median and } \\
\text { morphological } \\
\text { filering }\end{array}$ & DM - ASM & No & $\begin{array}{l}\text { Contour MD } \\
\text { Contour MaxD } \\
\text { Area OV }\end{array}$ & $\begin{array}{l}3.77(\approx 2.55 \mathrm{~mm}) \pm \\
1.3 \text { pixels } \\
6.25(\approx 4.18 \mathrm{~mm}) \pm \\
1.8 \text { pixels } \\
93 \% \pm 0.9 \%\end{array}$ & 10 images \\
\hline & Hodge [136] & 2006 & $3 \mathrm{D}$ & Yes & Median filter & DM - ASM & No & $\begin{array}{l}\text { Contour MD } \\
\text { Contour MAD } \\
\text { Contour MaxD } \\
\text { Volume VD }\end{array}$ & $\begin{array}{l}0.12 \pm 0.45 \mathrm{~mm} \\
1.09 \pm 0.49 \mathrm{~mm} \\
7.27 \pm 2.32 \mathrm{~mm} \\
0.22 \pm 4.58 \%\end{array}$ & 36 data sets \\
\hline & $\mathrm{Hu}[54]$ & 2002 & $3 \mathrm{D}$ & Yes & No & DM - Curve Fitting & No & $\begin{array}{l}\text { Contour MD } \\
\text { Contour MAD } \\
\text { Contour MaxD } \\
\text { Volume VD }\end{array}$ & $\begin{array}{l}(-) 0.2 \pm 0.28 \mathrm{~mm} \\
1.19 \pm 0.14 \mathrm{~mm} \\
7.01 \pm 1.04 \mathrm{~mm} \\
7.2 \pm 3.4 \%\end{array}$ & 5 data sets \\
\hline & Gong [90] & 2004 & $2 \mathrm{D}$ & Yes & No & DM - Curve Fitting & No & $\begin{array}{l}\text { Contour MD } \\
\text { Contour HD }\end{array}$ & $\begin{array}{l}1.36 \pm 0.58 \mathrm{~mm} \\
3.42 \pm 1.52 \mathrm{~mm}\end{array}$ & 125 images \\
\hline & Badiel [56] & 2006 & $2 \mathrm{D}$ & No & No & DM - Curve Fitting & No & $\begin{array}{l}\text { Area SN } \\
\text { Area AC } \\
\text { Contour MAD } \\
\text { Contour MaxD }\end{array}$ & $\begin{array}{l}97.4 \pm 1 \% \\
93.5 \pm 1.9 \% \\
0.67 \pm 0.18 \mathrm{~mm} \\
2.25 \pm 0.56 \mathrm{~mm}\end{array}$ & 17 images \\
\hline & Mahdavi[58] & 2011 & $3 \mathrm{D}$ & Yes & No & DM - Curve Fitting & No & Volume VE & $6.63 \pm 0.9 \%$ & 21 data sets \\
\hline
\end{tabular}


Table 6: Quantitative evaluations : prostate segmentation in TRUS imaging continued...

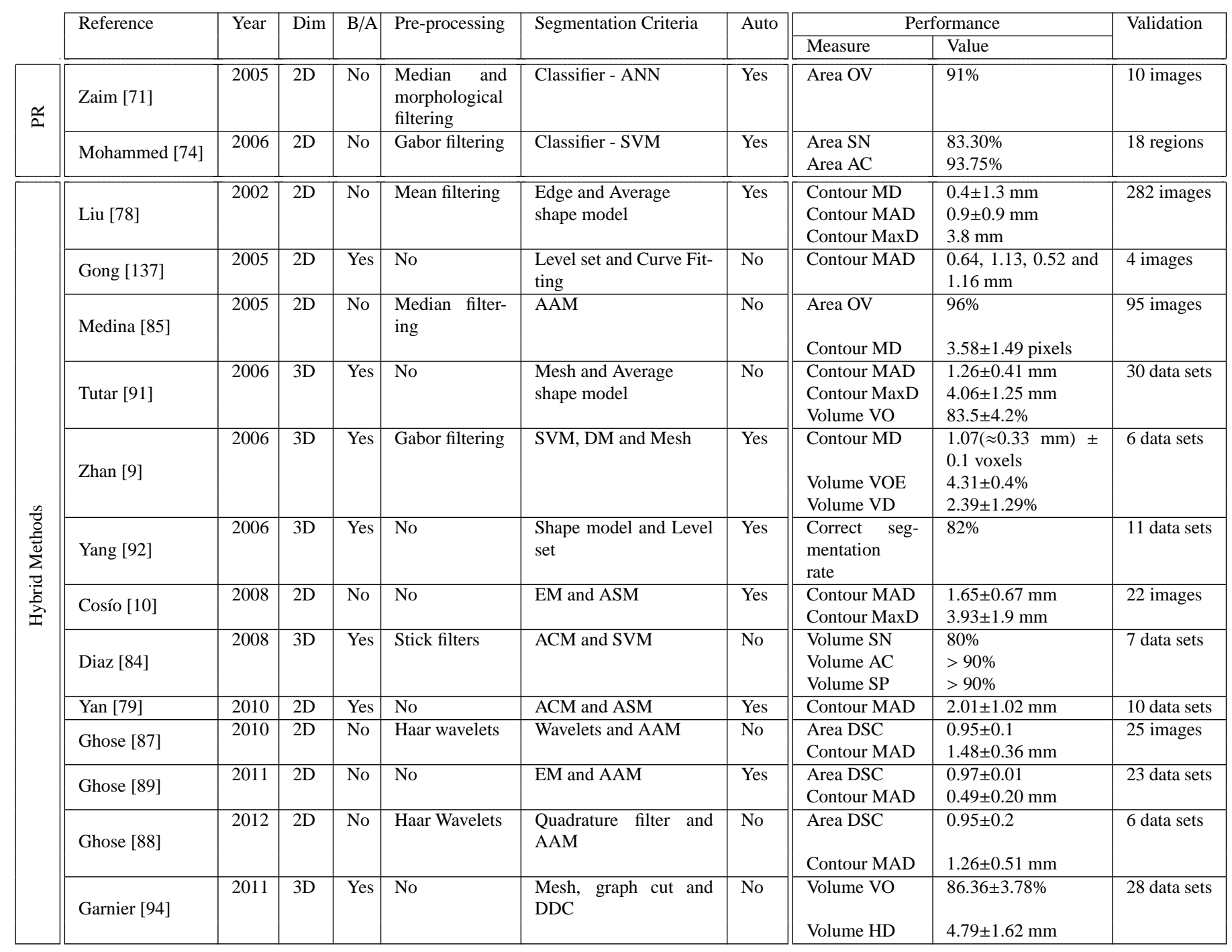


Table 7: Quantitative evaluations : prostate segmentation in MR images

\begin{tabular}{|c|c|c|c|c|c|c|c|c|c|c|}
\hline & \multirow[t]{2}{*}{ Reference } & \multirow[t]{2}{*}{ Year } & \multirow[t]{2}{*}{ Dim } & \multirow[t]{2}{*}{ In. } & \multirow[t]{2}{*}{ Pre-processing } & \multirow[t]{2}{*}{ Segmentation Criteria } & \multirow[t]{2}{*}{ Auto } & \multicolumn{2}{|c|}{ Performance } & \multirow[t]{2}{*}{ Validation } \\
\hline & & & & & & & & Measure & Value & \\
\hline \multirow{3}{*}{ 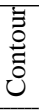 } & Samiee [20] & 2006 & $2 \mathrm{D}$ & Yes & Normalization & Edge Based & No & Area DSC & $0.9057 \pm 0.0014$ & 2 data sets \\
\hline & Flores-Tapia [21] & 2008 & $2 \mathrm{D}$ & Yes & Wavelets & Edge Based & No & Area DSC & $0.93 \pm 0.005$ & 19 images \\
\hline & Zhu [47] & 2007 & $3 \mathrm{D}$ & No & No & DM - ASM & No & RMSD & $5.4811 \pm 2.9 \mathrm{~mm}$ & 26 data sets \\
\hline \multirow{3}{*}{ 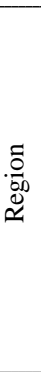 } & Klein [60] & 2008 & $3 \mathrm{D}$ & No & No & Atlas & Yes & Volume median DSC & 0.85 & 50 data sets \\
\hline & Langerak [63] & 2010 & $3 \mathrm{D}$ & No & No & Atlas & Yes & $\begin{array}{l}\text { Volume DSC error } \\
\text { Volume SN/SP error }\end{array}$ & $\begin{array}{l}0.0 .05 \\
0.05 \\
0.99\end{array}$ & $\begin{array}{ll}100 & \text { data } \\
\text { sets } & \end{array}$ \\
\hline & Dowling [62] & 2011 & $3 \mathrm{D}$ & No & $\begin{array}{l}\text { Bias field } \\
\text { correction, } \\
\text { histogram } \\
\text { equalization } \\
\text { and smoothing }\end{array}$ & Atlas & Yes & $\begin{array}{l}\text { Volume Median DSC } \\
\text { Area SD }\end{array}$ & $2.00 \pm 1.3 \mathrm{~mm}$ & 50 data sets \\
\hline \multirow{11}{*}{ 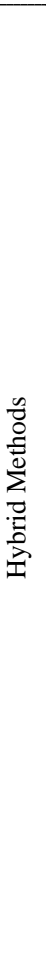 } & Allen [96] & 2006 & $3 \mathrm{D}$ & No & No & EM and DM & No & $\begin{array}{l}\text { Contour MAD } \\
\text { Volume VD }\end{array}$ & $\begin{array}{l}2.8 \pm 0.82 \mathrm{~mm} \\
6.5 \pm 5.4 \%\end{array}$ & 22 data sets \\
\hline & Martin [2] & 2008 & $3 \mathrm{D}$ & - & No & Atlas and DM & No & Mean error & $3.39 \pm 1.95 \mathrm{~mm}$ & 18 data sets \\
\hline & Makni [3] & 2009 & $3 \mathrm{D}$ & - & No & $\begin{array}{l}\text { DM and Bayes } \\
\text { Classifier }\end{array}$ & Yes & $\begin{array}{l}\text { Volume HD } \\
\text { Volume DSC }\end{array}$ & $\begin{array}{l}9.62 \mathrm{~mm} \\
0.90\end{array}$ & 12 data sets \\
\hline & Vikal [95] & 2009 & $3 \mathrm{D}$ & Yes & Stick filters & $\begin{array}{l}\text { Edge and Shape } \\
\text { guidance }\end{array}$ & No & $\begin{array}{l}\text { Contour MAD } \\
\text { Area DSC in mid } \\
\text { slice }\end{array}$ & $\begin{array}{l}2.0 \pm 0.6 \mathrm{~mm} \\
0.93 \pm 0.3\end{array}$ & 3 data sets \\
\hline & Liu [6] & 2009 & $2 \mathrm{D}$ & Yes & No & DM and Level set & Yes & Area DSC & $0.91 \pm 0.03$ & 10 data sets \\
\hline & Firjani [101] & 2010 & $2 \mathrm{D}$ & Yes & No & Intensity and shape & Yes & Area OE & $5.2 \pm 1.2 \%$ & 98 images \\
\hline & Firjani [102] & 2011 & $3 \mathrm{D}$ & Yes & No & Intensity and shape & Yes & Contour MD & $0.8 \pm 0.9 \mathrm{~mm}$ & 98 images \\
\hline & Martin [97] & 2010 & $3 \mathrm{D}$ & No & No & $\begin{array}{l}\text { Probabilistic Atlas } \\
\text { and DM }\end{array}$ & Yes & $\begin{array}{l}\text { Area SD } \\
\text { DSC } \\
\text { RMSD } \\
\text { MaxD } \\
\text { Sensitivity }\end{array}$ & $\begin{array}{l}2.41 \mathrm{~mm} \\
0.84 \\
1.97 \\
9.04 \\
0.86\end{array}$ & 36 data sets \\
\hline & Gao [104] & 2010 & $3 \mathrm{D}$ & No & No & $\begin{array}{l}\text { Shape and Edge } \\
\text { guidance for level sets }\end{array}$ & No & $\begin{array}{l}\text { Volume DSC } \\
\text { Volume HD } \\
\text { Volume DSC } \\
\text { Volume HD } \\
\end{array}$ & $\begin{array}{l}0.82 \pm 0.03 \\
10.22 \pm 4.03 \mathrm{~mm} \\
0.84 \pm 0.03 \\
8.10 \pm 1.50 \mathrm{~mm}\end{array}$ & $\begin{array}{l}15 \text { data sets } \\
15 \text { data sets } \\
13 \text { data sets } \\
13 \text { data sets }\end{array}$ \\
\hline & Toth [105] & 2011 & $3 \mathrm{D}$ & Yes & $\begin{array}{l}\text { Multiple ker- } \\
\text { nel Gaussian } \\
\text { filtering }\end{array}$ & $\begin{array}{l}\text { Shape and Edge } \\
\text { Based }\end{array}$ & Yes & Volumetric ratio & $1.05 \pm 0.21$ & 45 data sets \\
\hline & Toth [106] & 2011 & $3 \mathrm{D}$ & Yes & No & $\begin{array}{l}\text { Shape and Edge } \\
\text { Based }\end{array}$ & Yes & $\begin{array}{l}\text { Volume VO } \\
\text { Volume SN } \\
\text { Volume SP } \\
\text { Contour HD } \\
\text { Contour MAD } \\
\end{array}$ & $\begin{array}{l}0.7 \\
0.81 \\
0.99 \\
7 \mathrm{~mm} \\
5 \mathrm{~mm} \\
\end{array}$ & 32 data sets \\
\hline
\end{tabular}


Table 8: Quantitative evaluations : prostate segmentation in CT images

\begin{tabular}{|c|c|c|c|c|c|c|c|c|c|c|}
\hline & \multirow[t]{2}{*}{ Reference } & \multirow[t]{2}{*}{ Year } & \multirow[t]{2}{*}{ Dim } & \multirow[t]{2}{*}{ In. } & \multirow[t]{2}{*}{ Pre-processing } & \multirow{2}{*}{$\begin{array}{l}\text { Segmentation } \\
\text { Criteria }\end{array}$} & \multirow[t]{2}{*}{ Auto } & \multicolumn{2}{|c|}{ Performance } & \multirow[t]{2}{*}{ Validation } \\
\hline & & & & & & & & Measure & Value & \\
\hline \multirow{3}{*}{ 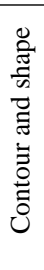 } & Knoll [33] & 1999 & $3 \mathrm{D}$ & - & Wavelets & $\overline{D M}-\mathrm{ACM}$ & Yes & $\begin{array}{l}\text { Contour MD } \\
\text { Volume VD } \\
\text { Volume AVD }\end{array}$ & $\begin{array}{l}2.48 \mathrm{~mm} \\
11.78 \% \\
9.5 \%\end{array}$ & 86 Images \\
\hline & Tang [48] & 2004 & $2 \mathrm{D}$ & - & No & DM - ASM & Yes & Contour MD & $2.44 \pm 1.24 \mathrm{~mm}$ & 5 images \\
\hline & Feng [49] & 2009 & $3 \mathrm{D}$ & - & No & DM - ASM & No & $\begin{array}{l}\text { Volume DSC } \\
\text { Area SD }\end{array}$ & $\begin{array}{l}90.5 \pm 4 \% \\
1.9 \pm 0.71 \mathrm{~mm} \\
\end{array}$ & 24 data sets \\
\hline \multirow{2}{*}{ 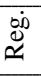 } & Costa [64] & 2010 & $3 \mathrm{D}$ & No & No & Atlas & Yes & Volume DSC & $0.564 \pm 0.192$ & 19 data sets \\
\hline & Costa [65] & 2011 & $3 \mathrm{D}$ & No & No & Atlas & Yes & Volume DSC maximum & 0.47 approx. & 24 data sets \\
\hline \multirow{12}{*}{ 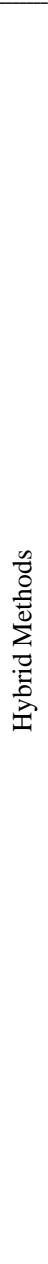 } & Freedman [109] & 2005 & $3 \mathrm{D}$ & - & No & S-R Mesh & & $\begin{array}{l}\text { Median volume VD } \\
\text { Volume VCD } \\
\text { Area SD }\end{array}$ & $\begin{array}{l}89 \%, 81 \%, 85 \% \\
3.17 \text { pix }(\approx 2.94 \\
\mathrm{mm}), 5.29 \text { pix }(\approx \\
4.91 \mathrm{~mm}), \quad 4.07 \\
\text { pix }(\approx 3.78 \mathrm{~mm}) \\
\begin{array}{l}0.57, \quad 1.02, \quad 0.83 \\
\text { pix }\end{array}\end{array}$ & $\begin{array}{l}48 \text { data sets } \\
\text { of } 3 \text { pa- } \\
\text { tients }\end{array}$ \\
\hline & Rousson [110] & 2005 & $3 \mathrm{D}$ & - & No & S-R Level set & No & $\begin{array}{l}\text { Area OV } \\
\text { FP } \\
\text { Volume VCD } \\
\text { Area SD }\end{array}$ & $\begin{array}{l}84 \% \\
21 \% \\
5.2 \mathrm{~mm} \\
4.2 \mathrm{~mm}\end{array}$ & 16 images \\
\hline & Davis [111] & 2005 & $3 \mathrm{D}$ & - & No & $\begin{array}{l}\text { DM and registra- } \\
\text { tion }\end{array}$ & Yes & Volume DSC & $0.82 \pm 0.06$ & 40 dataset \\
\hline & Costa [113] & 2007 & $3 \mathrm{D}$ & - & No & S-R Level set & Yes & $\begin{array}{l}\text { Area SN } \\
\text { Area AC }\end{array}$ & $\begin{array}{l}75 \% \\
80 \%\end{array}$ & 16 data sets \\
\hline & Feng [119] & 2010 & $3 \mathrm{D}$ & - & No & $\begin{array}{l}\text { ASM and appear- } \\
\text { ance model }\end{array}$ & No & $\begin{array}{l}\text { Volume DSC } \\
\text { Area SD }\end{array}$ & $\begin{array}{l}92.4 \\
1.47 \mathrm{~mm}\end{array}$ & 24 data sets \\
\hline & Song [114] & 2009 & $3 \mathrm{D}$ & - & No & $\begin{array}{l}\text { ACM, Mesh and } \\
\text { Graph Cut }\end{array}$ & No & $\begin{array}{l}\text { Volume VDe } \\
\text { Volume FP } \\
\text { Area SD }\end{array}$ & $\begin{array}{l}85.2 \% \\
13.60 \% \\
1.38 \pm 1.08 \mathrm{~mm}\end{array}$ & 21 data sets \\
\hline & Chen [117] & 2009 & $3 \mathrm{D}$ & - & No & S-R Level set & No & Volume VS & $93.20 \%$ & 15 dataset \\
\hline & Chen [118] & 2009 & $2 \mathrm{D}$ & - & No & S-R Level set & Yes & DSC & $0.91 \pm 0.90$ & 10 images \\
\hline & $\operatorname{Li}[120]$ & 2011 & $3 \mathrm{D}$ & - & No & $\begin{array}{l}\text { Classifier and } \\
\text { level sets }\end{array}$ & Yes & $\begin{array}{l}\text { DSC } \\
\text { Area SD }\end{array}$ & $\begin{array}{l}0.908 \\
1.40 \mathrm{~mm}\end{array}$ & 11 data sets \\
\hline & Liao [121] & 2011 & $3 \mathrm{D}$ & - & No & $\begin{array}{l}\text { Classifier } \\
\text { registration }\end{array}$ & Yes & Volume DSC & $0.89 \pm 0.02$ & 10 data sets \\
\hline & $\mathrm{Lu}[122]$ & 2011 & $3 \mathrm{D}$ & - & No & $\begin{array}{l}\text { S-R Level set and } \\
\text { registration }\end{array}$ & Yes & $\begin{array}{l}\text { Contour MAD } \\
\text { Contour HD }\end{array}$ & $\begin{array}{l}1.96 \pm 0.48 \mathrm{~mm} \\
2.83 \pm 0.76 \mathrm{~mm}\end{array}$ & 32 data sets \\
\hline & Chen [123] & 2011 & $3 \mathrm{D}$ & - & $\begin{array}{l}\text { Median and } \\
\text { morphological } \\
\text { filtering }\end{array}$ & $\begin{array}{l}\text { Shape and } \\
\text { anatomy con- } \\
\text { strained intensity } \\
\text { based registration }\end{array}$ & Yes & $\begin{array}{l}\text { Volume TP } \\
\text { Volume FP } \\
\text { Area SD }\end{array}$ & $\begin{array}{l}0.84 \\
\\
\\
0.13 \\
1.1 \mathrm{~mm}\end{array}$ & $\begin{array}{l}185 \text { data } \\
\text { sets }\end{array}$ \\
\hline
\end{tabular}

